\title{
Automatic Semantic Header Generator
}

\author{
Bipin C. DESAI, Sami S. HADDAD \\ Department of Computer Science, \\ Concordia University, \\ Montreal, H3G 1M8, CANADA
}

December 13, 1998

\begin{abstract}
As the amount of information and the number of Internet users grow, the problem of indexing and retrieval of electronic information resources becomes more critical. The existing search systems tend to generate misses and false hits due to the fact that they attempt to match the specified search terms without context in the target information resource. The COncordia INdexing and DIscovery system is an indexing system. It is a powerful means of helping users locate documents, software, and other types of data among large repositories. In environments that contain many different types of data, content indexing requires type-specific processing to extract information effectively. The Semantic Header, which is proposed by Desai [11], contains the semantic contents of information resources. It provides a useful tool in searching for a document based on a number of commonly used criteria. The information from the semantic header could be used by the search system to help locate appropriate documents with minimum effort. This paper introduces an automatic tool for the extraction and storage of some of the meta-information in a Semantic Header and an automatic text classification scheme.
\end{abstract}

\section{Introduction}

Rapid growth in data volume, user base and data diversity render Internet-accessible information increasingly difficult to use effectively. At this time, a number of information sources, both public and private, are available on the Internet. They include text, computer programs, books, electronic journals, newspapers, organisational, local and national directories of various types, sound and voice recordings, images, video clips, scientific data, and private information services such as price lists and quotations, databases of products and services, and speciality newsletters [12]. There is a need for an automated search system that allows easy search for and access to relevant resources available on the Internet. Proper 
functioning of this system will require a proper indexing of the available information. Therefore, secondary information must be extracted and used as an index to the available primary resource. Building this index requires information extraction methods tailored to each specific environment. The semantics of the files in which the primary resource is stored will be exploited in order to extract and summarise the relevant information that will support the resource discovery. To do this, the primary file type should be identified and then the type specific selection and extraction methods are applied to the file.

It is envisioned that regional and/or specialised databases will be created to maintain archives of the cover pages (or Semantic Headers). These databases could be searched by cooperating distributed expert systems to help users in locating pertinent documents. Such a system is currently under development at Concordia University and is called Concordia INdexing and DIscovery system, or CINDI.

CINDI, a system under development at Concordia University, provides a mechanism to register, search and manage the meta-information, with the help of an easy to use graphical user interface. This meta-information, which is described in section 2, is the Semantic Header, that is stored in the CINDI system. CINDI tries to avoid problems caused by differences in semantics and representation as well as incomplete and incorrect data cataloguing. It also tries to avoid the problems caused by the difference in index terms. This meta-information could be entered either by the primary resource provider or by the Automatic Semantic Header Generator (ASHG). ASHG, a software that generates some meta-information of the submitted document, assists the user in this process. This thesis introduces ASHG, which aims at saving the primary resource provider's time by automatically generating and extracting part of the meta-information (Semantic Header) of the document and classifying the resource under a list of subject headings. As the provider helps in this process by verifying and correcting the Semantic Header entry, there is the potential for its accuracy is high.

\section{$1.1 \quad$ Organisation of the paper}

This paper is organised as follows. In section 2, we will introduce the CINDI system. Section 3 describes information retrieval, its history and some of the algorithms used in that field. Automatic text retrieval, natural language processing and text classification is also discussed in section 3. At the end of section 3, we describe some retrieval and information extraction systems. Section 4 covers the Thesaurus used and how it is built. Section 5 describes the Automatic Semantic Header Generator, or ASHG. This section covers the basic subparts used by it as the type recognition, and the extractors. In section 6 , we test and compare the classification of our generated index with the ones produced by cataloguers or the document's author's opinion. Finally in section 7, we draw our conclusion.

\section{The CINDI system}

The current practice in most research institutes, universities and business organisations to interconnect their computing facilities using a digital network is the accepted method of sharing resources. Such networks, in turn, are interconnected allowing information to be exchanged across networks using appropriate data transfer protocols. 
There is a need for the development of a system which allows easy search for and access to resources available on the Internet. Solving the problem of fast, efficient and easy access to the documents can be started by building a standard index structure and building a bibliographic system using standardised control definitions and terms. Such definitions could be built into the knowledge-base of an expert system based index entry and search interface. The purpose of indices and bibliographies (secondary information) is to catalogue the primary information and allow easy access to it.

Preparing the primary source's meta or secondary information requires finding the primary source, identifying it as to its subject, title, author, keywords, abstract, etc. Since it is to be used by many users, it has to be accurate, easy to use and properly classified. Attempts to provide easy search of relevant documents has lead to a number of systems including WAIS, and more recently a number of Spiders, Worms and other creepy crawlers [9, 20, $28,39,65,60,68,69,70]$. However, the problem with many of these tools is that their selectivity of documents is often poor [12]. The chances of getting inappropriate documents and missing relevant information because of poor choice of search terms is large. These problems are addressed by CINDI, which provides a mechanism to register, manage and search the bibliographic information.

\subsection{Overview of CINDI}

The overall CINDI system uses knowledge bases and expert sub-systems to help the user in the registering and the search processes. CINDI standardises the terms. The index generation and maintenance sub-system uses CINDI's thesaurus to help the provider of the resource select correct terms for items such as subject, sub-subject and keywords. Similarly, another expert sub-system is used to help the user in the search for appropriate information resources [11].

\subsubsection{The Semantic Header}

For cataloguing and searching, CINDI uses a meta-data description called a Semantic Header to describe an information resource. The Semantic Header includes those elements that are most often used in the search for an information resource. Since the majority of searches begin with a title, name of the authors (70\%), subject and sub-subject (50\%) [27], CINDI requires the entry for these elements in the Semantic Header. Similarly, the abstract and annotations are relevant in deciding whether or not a resource is useful, so they are included too. $[56,12]$. A brief descripton of the semantic header elements follows:

1. Title, Alt-title: The title field contains the name of the resource that is given by the creator(s). The alternate title field is used to indicate a secondary title of the resource.

2. Subject: The subject and sub-subjects of the resource are indicated in the next field which is a repeating group. This field contains a list of possible subject classifications of the resource.

3. Language, Character Set: The character set and the language are the ones used in resource. 
4. Author and other responsible agents: The role of the person associated with the document, for instance, author, editor, and compiler. This includes fields such as name, postal address, telephone number, fax number, and email address.

5. Keyword: This field contains a list of keywords mentioned in the resource.

6. Identifier: The identifiers for the document. Example of identifiers are, ISBN(International Standard Book Number), URL (Universal Resource Locator) of the document. This is a multi-valued slot in case the document is available in many formats or is electronically stored at more than one site.

7. Date: The date on which the document was created, catalogued, and the date on which the document will expire, if any.

8. Version: The version number, and the version number being superseded, if any, are given in these elements.

9. Classification: The legal, security or other type of classification of the document. For each, nature of classification is specified.

10. Coverage: It indicates the targeted audience of the document or it may indicate cultural and temporal aspect of the document's content.

11. System Requirements: The document being an electronic one requires certain system requirements for it to be displayed or used. The components are the hardware, the software or the network and for each the minimum needs.

12. Genre: It is used to describe the physical or electronic format of the resource. It consists of a domain and the corresponding value or size of the resource.

13. Source and Reference: The Source indicates the documents being referenced or which were required in its preparation. It could also be the main component for which the current document is an addendum or attachment.

14. Cost: In case of a resource accessible for a fee, the cost of accessing it is given.

15. Abstract: The abstract of the document is either provided by the author or by ASHG.

16. Annotations: Annotations put in by readers of the document.

17. User ID, Password: A Provider ID of at least six characters and a password of four to eight characters. More than one semantic header by the same provider can have the same ID and password.

Next, the Semantic Header Database system is described. 


\subsection{The Semantic Header Database System}

The index entries registered by a provider of a resource is stored in a distributed database system (SHDDB). From the point of view of the users of the system, the underlying database may be considered to be a monolithic system. In reality, it would be distributed and replicated allowing for reliable and failure-tolerant operations. The interface hides the distributed and replicated nature of the database. The distribution is based on subject areas and as such the database is considered to be horizontally partitioned [10]. It is envisaged that the database on different subjects will be maintained at different nodes of the Internet. The locations of such nodes need only be known by the intrinsic interface. A database catalog would be used to distribute this information. However, this catalog itself could be distributed and replicated as is done for distributed database systems.

The Semantic Header information entered by the provider of the resource using a graphical interface is relayed from the user's workstation by a client process to the database server process at one of the nodes of the SHDDB. The node is chosen based on its proximity to the workstation or on the subject of the index record. On receipt of the information, the server verifies the correctness and authenticity of the information and on finding everything in order, sends an acknowledgment to the client. The server node is responsible for locating the partitions of the SHDDB where the entry should be stored and forwards the replicated information to appropriate nodes. For example, the semantic header entry would be part of the SHDDB for subjects Computer Science and Library Studies. Similarly the database server process is responsible for providing the catalogue information for the search system. In this way the various sites of the database work in a cooperating mode to maintain consistency of the replicated portion. The replicated nature of the database also ensures distribution of load and ensures continued access to the bibliography when one or more sites are temporarily nonfunctional.

\subsection{The CINDI's Search System}

CINDI guides the user in entering the various search items in a graphical interface similar to the one used by the index entry system. The search system also uses a graphical interface and a client process. Once the user has entered a search request, the client process communicates with the nearest SHDDB catalogue to determine the appropriate site of the SHDDB database. Subsequently, the client process communicates with this database and retrieves one or more semantic headers. The result of the query could than be collected and sent to the user's workstation. The contents of these headers are displayed, on demand, to the user who may decide to access one or more of the actual resources. It may happen that the item in question may be available from a number of sources. In such a case the best source is chosen based on optimum costs. The client process would attempt to use appropriate hardware/software to retrieve the selected resources [12].

\section{Information Retrieval Overview}

Information Retrieval (IR) is concerned with the representation, storage, organisation and accessing of information. The first step in the retrieval process is for the user to state the 
information needed. This has to be done in a format that enables the IR system to understand it and to act on it [19]. To facilitate the task of finding items of interest, libraries and information centers provide information users with a variety of auxiliary aids. Each incoming item is analysed and appropriate descriptions are chosen to reflect the information content of the item. Retrieval effectiveness is typically measured by two metrics, precision, which is the percentage of the retrieved documents that are relevant to the information need, and recall, which is the percentage of relevant documents in the collection that are retrieved. [19]. Indexing is the basis for retrieving documents that are relevant to the user's need [34]. Building an accurate representation of a document, which would increase precision, is one of CINDI's main concerns. Compact descriptions of a document's index may increase the efficiency of matching and the effectiveness of classifying textual material as relevant or nonrelevant. Document retrieval imposes conflicting normalising and accurate demands [34]. As a result, variations in indexing that increase precision usually decrease recall, and vice versa. The fundamental goal is to increase both. There are numerous types of indexing languages. One which uses the same terms found in the document and another which is limited to those from a controlled languages [34].

In this section, we will discuss the history of information retrieval, automatic document indexing or representation, algorithms used by the IR community, natural language processing, the automatic sentence extraction, abstract selection and the text classification. We will also be briefly portraying Salton's SMART retrieval system, Oracle's ConText, Nordic and Harvest's Essence information retrieval and extraction systems.

\subsection{Information Retrieval Background}

Tests of indexing languages have shown that indexing documents by individual terms corresponding to words or word stems produces results that are at least as good as those produced when indexing by controlled vocabularies [34]. Luhn[36] used frequency counts of words in the document text to determine which words were sufficiently significant to represent the document. The use of statistical information about distributions of words in documents was further exploited by Maron and Kuhn [37] and Stiles [58] who obtained statistical associations between keywords. Statistical Document Retrieval methods assign higher numeric weights to terms showing evidence of being good content indicators, causing them to have greater influence on the ranking of the documents. The number of occurrences of a term in a document as a whole may be taken into account, when computing the influence of the term. Evidence also suggests that combining single terms into compound terms may be useful[34].

\subsection{Developments in Automatic Text Retrieval}

In conventional information retrieval, the stored records are normally identified by sets of keywords or phrases known as index terms. Requests for information are typically expressed by Boolean combinations of index terms, consisting of search terms interrelated by the Boolean operators and, or, and not. The retrieval system is then designed to select those stored items that are identified by the exact combination of search terms specified in the available queries. The terms characterising the stored texts may be assigned manually by trained personnel, or automatic indexing methods may be used to handle the term assignment. Refinements have been introduced into the Boolean processing environment. They allowed the terms assigned 
to documents to carry term weights. When term weights were introduced, they were called the fuzzy-set retrieval model.

\subsection{Algorithms used by the IR community}

The IR community's main concern is how to select significant words and phrases from a document that best describe the document or set of documents [36, 15]. Automatic summarisation of full documents generates a condensed version of the document[7]. The condensed version serves as an executive summary, which contains indicative information of the document's content. Automatic summarisation of full documents ascertains the relative importance of the material and generates coherent output[7]. The IR community has tried to automatically find significant words in documents and understand the content or meaning of the document. The following subsections discuss some of the main ideas that make up the core of our system.

Luhn's ideas: Luhn assumes that frequency data can be used in extracting words and sentences that represent a document [36]. He ranked the words in the decreasing frequency of occurrence. After plotting the graph of frequency related to rank, he found that the curve was similar to the hyperbolic. This is in accordance with Zipf's law which states that the product of the frequency of use of words and the rank is approximately constant. He then excludes the non-significant words and the very high frequency words. Luhn also used this method to devise a method for automatic abstracting. He went on to develop a numerical measure of significance for sentences based on the number of significant and non-significant words in each portion of the sentence. Sentences were ranked according to their numerical score and only the highest ones would be included in the abstract.

C.J.van Rijsbergen's attempt: The document's representation aimed by Rijsbergen [46] consisted simply of a list of class names, each name representing a class of words occurring in the total input text. A document was indexed by a name if one of its significant words occurred as a member of that class. Such system consists of 3 parts:

1. Removal of high frequency words

2. Suffix stripping

3. Detecting equivalent stems

If two words have the same underlying stem, then they probably refer to the same concept and they should be indexed as such. It is inevitable that a processing system such as this will produce errors. Fortunately, experiments have shown that the error rate tends to be of the order of 5 per cent [2]. Lovins [35] using a slightly different approach to stemming also quotes errors of the same order of magnitude. The final output would be a set of classes, one for each stem detected. A class name is assigned to a document if one of its members occurs as a significant word in the document. 
Bayesian network: All IR systems draw conclusions about the content of a document by examining some representation of that document. An automated system of indexing in such an approach bases its conclusions about the document on the evidence of computable document features, such as the presence or absence of particular words and phrases [19]. A Bayesian network is a directed acyclic graph in which each node represents a random variable, that is a set of mutually exclusive and collectively exhaustive propositions. Each set of arcs into a node represents a probabilistic dependence between the node and its parents (the nodes at the other ends of the incoming arcs). A Bayesian network represents, through its structure, the conditional independence relations among the variables in the network. These independence relations provide a framework within which to acquire probabilistic information. A Bayesian network represents beliefs and knowledge about a particular class of situations. Given a Bayesian network for a class of situations and evidence about a particular situation in that class, conclusions about the document and document's relevant topics can be drawn [19]. The advantages that Bayesian networks bring to the IR task include an intuitive representation of uncertain relationships and a set of efficient inference algorithms. Robert Fung and Brendan Del Favero [19] have used a probabilistic IR architecture that assists users who have fixed information needs in routing large amounts of material. Towards these goals, they have developed and implemented a system that allows a user to specify the topics of interest (i.e., information need), the quantitative and qualitative relationships among the topics, the document features, such as the presence or absence of particular words and phrases, and the quantitative relationships between these features and the topics. [19].

The Vector Model: In the vector space model, documents are identified by sets of attributes, or terms. Instead of assuming that all terms are equally important, the system uses term weighting. The vector processing model offers simple, parallel treatments for both queries and documents. Extensions to the vector and Boolean models have been proposed including a generalised vector space model based on an orthogonal vector space. Another common retrieval model is the extended Boolean system which accommodates term weights assigned to both query and document terms as well as strictness indicators. The extended system thus covers vector processing, Boolean, and fuzzy set retrieval in a common framework, and it produces a vastly improved retrieval performance over simple Boolean operations.

The Probabilistic Model: The probabilistic retrieval model differs from those previously discussed. It represents an attempt to set the retrieval problem on a theoretical foundation. In the classical probabilistic models, the needed term probability is estimated by accumulating a number of user queries containing a term and determining the proportion of time a document is found relevant to the respective queries. Alternatively, a fixed query is considered and an attempt to determine the probability of an arbitrary document containing a query term will be judged relevant. The probabilistic retrieval approach accommodates a large number of different phenomena about terms and documents as part of the probabilistic estimation process. This includes term co-occurence information, term relationships derived from dictionaries and thesauruses, and prior knowledge about the occurrence distributions of terms. 


\subsubsection{Limitations of the Traditional Approaches}

Traditional approaches to information retrieval use keyword searches and statistical techniques to retrieve relevant documents (e.g., [61, 53]). Statistical techniques take advantage of large document collections to automatically identify words that are useful indexing terms. However, word-based techniques have several limitations:

- Synonymy: Different words and phrases can express the same concept.

- Polysemy: Words can have multiple meanings [38].

- Anaphora: is a phenomenon of abbreviated subsequent reference to refer back to an entity introduced with more descriptive phrasing earlier by using a lexically and semantically abbreviated form [57]. It is used to make language more concise and avoid repetition and the most common manifestation of this is in the use of pronouns. For example in the following passage the anaphoric reference their refers to the earlier target computers:

Computers are often mixed up with questions about their impact on ...

- Phrases: Some words are good indexing terms only in specific phrases.

- Local Context: Some words and phrases are good indexing terms only in specific local contexts.

- Global Context: Some documents do not contain any words or phrases that are good indexing terms.

\subsubsection{Enhancing the document representation}

The conventional wisdom is that the keyword-type systems, where the information items are represented by sets of manually or automatically chosen index terms, have run their course. Most keywords are believed to be ambiguous and are often poorly represented by small collections of individual terms [54]. It is therefore widely believed that the keyword approach is not adequate for text content representation in information retrieval. By extension, the identification of text content by weighted term sets may also be unacceptable. Quoting from Blair: [6]

No number of brute linguistic facts (word statistics) can be added up to give us the meaning of a text, where the meaning of a text would include such things as its subject, intellectual content, context, use, purpose, or links to other documents.

The available experimental evidence indicates that the use of abstracts in addition to titles brings substantial advantages in retrieval effectiveness. However, the additional utilisation of full texts of the documents appears to produce very little improvement over titles and abstracts alone in most subject areas [50]. This is one of the main reasons why the abstract is included in the Semantic Header. 


\subsection{Natural Language Processing in IR}

\subsubsection{Progress of Natural Language Processing in IR}

Information retrieval systems locate documents that are typically retrieved as a ranked list, where the ranking is based on estimations of relevance [5]. Lexical ambiguity is a pervasive problem in natural language processing, and previous literature divides it into two types: syntactic and semantic [29]. For Natural Language Processing, or NLP, lexical processing operates at the single word level and it involves identifying words and determining their grammatical classes or parts of speech so that higher levels of language analysis can take place [57]. This usually consists of looking up a dictionary or lexicon, essentially a list of known words and their legitimate morphological variants. Ideally, lexical processing determines one base form for each word. The main sources of structural syntactic ambiguity in English are the attachment of prepositional phrases, the construction of nominal compounds and the scope of coordination and conjunction.

The semantic level of language analysis is concerned with meaning and focuses on broad questions like what type of knowledge representation framework should be used [57]. On another level, there are semantic constraints on what should make semantically sensible natural language statements. The semantic level language analysis should be able to analyse grammatically parsed text into a knowledge representation. This is because a sentence may have a number of semantic interpretations, possibly arising from a number of syntactic interpretations, and as many of these should be eliminated. The difficulty with semantic processing is that all the properties of every object and the legitimate arguments of all verbs must be known [57]. As a possible remedy to this problem, huge knowledge base could be built. Detecting anaphora and resolving references would improve the understanding of a text. Even so, detecting anaphora is often difficult as there are no indicator phrases or terms. Some words are potentially anaphoric but not always so and anaphoric references can include many constructs. Although Liddy [13] lists almost 150 words which could be indicators of an anaphoric construct, the problem of reliably resolving anaphora still remains. It is important to note that fully-fledged NLP is being used in information retrieval [57]. This has led to the emergence of the application known as conceptual information retrieval. There, once the user requests information, he/she is given the information directly, instead of just receiving its reference.

Lexical level language processing in information retrieval The simplest applications of NLP to information retrieval have been at the word level. Indexing based on some normalised or derived form of individual words occurs in the input [57]. An alternative to the popular stemming and conflation procedures would involve determining the base forms of words from a lexicon lookup. Building such a lexicon is expensive considering its marginal improvements over mechanical stemming. For those reasons, the idea has never really been pursued. However, lexical level language analysis has had a surge of interest recently with the increased availability of machine-readable dictionaries (MRDs)[57]. Its obvious use is to index by word senses rather than by word base forms. In information retrieval experiments, indexing by word senses using MRDs initially gave disappointing results in terms of retrieval effectiveness [57]. Because of this, researchers believe that it may not be necessary to deter- 
mine the single correct sense of a word. Instead a sufficient understanding that allows one to rule out unlikely senses and to weigh likely senses highly, Krovetz and Croft stressed the importance of the word senses, that will provide a significant separation between relevant and non-relevant documents [29]. They mentioned that word ambiguity and the use of related or synonym words are two problems that arise when using words to represent the content of a document.

Syntactic level language processing in information retrieval NLP techniques have been used to help index texts by elements more complex than word forms. Syntactic analysis can be used to analyse text in order to determine the boundaries of noun phrases which could then be used as internal representations. Indexing texts on a noun phrase basis using NLP techniques was done in the IOTA system [8]. One major problem of indexing by noun phrase units is the variety of ways of representing a complex concept in natural language. Three approaches have addressed the issue of ambiguity in syntactic analysis of texts for indexing purposes: ignoring ambiguity, normalising the identified phrases or indexing by structures which incorporate the ambiguities. Ignoring the ambiguity allows texts to be indexed by phrases taken directly from the text. A large amount of work in this area has been done by Salton and others at Cornell University [55]. Normalising indexing phrases from texts and from queries into some standard form is used in the CLARIT project at Carnegie Mellon university [18]. A first order thesaurus for a domain, essentially a phrase list, is first generated automatically. Input texts are parsed and candidate noun phrases are identified. These are then compared to the thesaurus. They are classified as either:

1. exact (identical to some phrase in the list),

2. general (terms are constituents of those in the list), or

3. novel (new terms not on the list).

This approach always uses terms from the list as the indexing units and thus always yields the same syntactic form for a concept which could have been expressed in a number of different ways [18]. Encoding the ambiguity in some structure and allowing the retrieval operation to make allowances for this, handles the syntactic ambiguity in syntactically based indexing.

Semantic level language processing in information retrieval Any piece of text which contains information essentially consists of a description of objects and actions on those objects. A number of conceptual information retrieval systems are described in the literature: SCISOR [23], RESEARCHER [32] and OpEd[1]. SCISOR [23] parses and analyses input stories into a knowledge base and then it answers users' questions about the content. RESEARCHER operates in the domain of US patent applications. Trying to resolve outstanding ambiguities, RESEARCHER uses limited semantics to resolve syntactic ambiguity and then uses the knowledge assimilated from the whole of the patent application it is processing [32]. $\mathrm{OpEd}$ is an editorial comprehension and question answering system which answers questions about beliefs, belief relationships and goals of those who have made arguments in the input texts [1]. 


\subsection{Automatic Sentence Extraction used in Title and Abstract selection}

Text processing methods based on a determination of term or sentence importance have been used not only for indexing but also for automatic abstracting purposes [52]. It was hypothesised that an extract of a document, that is a selection of significant sentences can serve as an abstract. This hypothesis concerning the substitutability of extracts for abstracts has been discussed in [15]. To achieve this, each sentence in the source text is scored according to some measure of importance, and the best rated sentences are selected [59]. Ideally, given a document represented as natural language text, one would like to construct a coherent well written abstract that informs the readers of the contents of the original, or at least indicates whether the full version may be of interest to the reader. A useful first step in the automatic or semi-automatic generation of abstracts from source texts is the selection of a small number of sentences, which are deemed to be important for purposes of content representation, from the source text [59]. The extraction methods used over the years start with a calculation of word and sentence significance, similar in spirit to the computation of the term weights. Criteria for the selection of important terms may be positional (the term's location in the document), semantic, or pragmatic (a system which would consider proper names as highly significant). Statistical term weights may be also criteria in selecting important terms. Since the frequency criteria are not very reliable, additional criteria should be used such as contextual inference (the word location or the presence of cue words), and syntactic coherence criteria [36, 16, 15, 49, 14, 4, 42, 43].

Kupiec et al.[30] describe a classification task on the basis of a corpus of technical papers with summaries written by professional abstractors. Their system identifies sentences in the text which also occur in the summary. Then it acquires a model of the abstract-worthiness of a sentence as a combination of a limited number of properties of that sentence. These properties include the sentence location in the document, the sentence length and the presence of thematic words in the sentence.

Simone H. Teufel and Marc Moens [59] report on a replication of Kupiec's experiment with different data. Summaries for their documents were not written by professional abstractors, but by the authors themselves. This produced fewer alignable sentences to train on. They used alternative meaningful sentences (selected by a human judge) as training and evaluation material, because this has advantages for the subsequent automatic generation of more flexible abstracts. They employed five different heuristics: four of the methods used by Kupeic et al as well as the title method described below. Kupeic et al's methods were the cue phrase method, location method, sentence length method and thematic word method.

1. Cue phrase method: it seeks to filter out meta-discourse from subject matter. Cue phrases were manually classified into five classes. This corresponds to the likely-hood of a sentence containing the given cue to be included in the summary. A score of minus one means very unlikely to be included in the summary, whereas a score of plus three means very likely to be included in the summary.

2. Location method: Paragraphs at the start and at the end are more likely to contain material that is useful for a summary. These paragraphs tend to include crucial infor- 
mation. Simone et al's algorithm assigns non-zero values only to sentences which are in document peripheral sections. Sentences in the middle receive a zero score.

3. Sentence length method: All sentences under a certain length (fifteen tokens including punctuation are given a score of zero. All sentences above that criterion are assigned a score of one.

4. Thematic word method: It identifies key words that are characteristic for the contents of the document. The top ten scoring words are chosen as the thematic words. Sentence scores are then computed as a weighted count of thematic words in that sentence.

5. Title method: Words occurring in the title are good candidates for document specific concepts. Simone et al. also experimented by taking into accounts words occurring in the headings. Better results were generated using title words only [59].

\subsection{Text Classification or Categorisation}

An important step in building up the document database of a full text retrieval system is to classify each document under one or more classes according to the topical domains that the document discusses. This is commonly referred to as classification. Automatic classification has two major components:[24] The classification scheme, which defines the available classes under which a document can be classified and their inter-relationships, and the classification algorithm, which defines the rules and procedures for assigning a document to one or more classes. Text categorisation systems assign predefined category labels to texts. For example, a text categorisation system for computer science might use categories such as operating systems, programming languages, AI or information retrieval [47]. Text Categorisation are typically applied to static databases [47].

Wong. Kan and Young presented an automatic classification approach called ACTION [24]. The key idea behind it is a scheme for measuring the significance of each keyword in a given document. That scheme takes into account not only the occurrence frequency of a keyword, but also the logical relationship between the available classes.

The relevancy signatures algorithm [48] uses linguistic phrases, the augmented relevancy signatures algorithm uses phrases and local context, and the case-based text classification algorithm uses larger pieces of context. These three algorithms were evaluated and the results suggested that information extraction techniques can support high-precision text classification. In general, using more extracted information improves performance. There have been approaches using knowledge bases relying on a domain-specific dictionary to drive the information extraction system [48]. It seems reasonable to believe that we could produce accurate classifications if we could actually understand the documents. However, natural language understanding is an expensive endeavour that can strain computational resources. Thus, some researches have turned their attention to information extraction that extracts specific types of information from a document [48]. The main advantage of this approach is that portions of a text that are not relevant to the domain can be effectively ignored. Since the system is only concerned with the domain-specific portions of the text, some of the most 
difficult problems in NLP are simplified. As a result, information extraction is a practical and feasible technology that has achieved success in the last few years [33, 48].

Edmundson [17] describes new methods of automatically extracting sentences from documents for screening purposes. His method describes the sentence significance, the high content words previously described and three additional components: pragmatic words (cue words), words found in the title and the headings, and the structural indicators (sentence location). An attempt was made by Edmundson to classify eligible sentences as to qualitative degree of extract-worthiness. In practice, however, it did not prove satisfactory for sentence selection. The principles he followed in devising the guide to the development of automatic extracting methods so as to yield close approximations to target extracts were:

1. Detect and use all content and format clues to the relative importance of sentences that were originally provided by the author, editor or printer.

2. Employ a system of reward weights for desired sentences and penalty for undesired sentences.

3. Employ a system of parameters that can be varied to permit different specifications for extracts.

4. Employ a method that is a function of several linguistic factors (syntactic, semantic, statistical locational, etc.).

Thus, the four basic methods Edmundson used in his automatic extracting system are the Cue, Key, Title and location methods. Clearly, there are extracting clues that have not been exploited-in captions of figures and tables, in footnotes and references.

\subsection{Retrieval and Information extraction systems}

\subsubsection{The SMART Retrieval System}

The SMART system is a sophisticated text retrieval tool based on storing all information terms in a vector of terms. In principle, the terms might be chosen from a controlled vocabulary list or a thesaurus [55]. A summary of the results after applying the SMART system shows that abstract processing with phrase and synonym recognition had the best results. Next most effective were the results that were drawn from using weighted word stem matching and statistical word associations using abstracts for analysis purposes. The less effective results were upon matching logical word stem and disregarding term weights. The least effective results were when only document titles were used for analysis purposes.

\subsubsection{Oracle ConText-Text Management System}

The Oracle7 ConText option is a fully integrated text management solution that enables users to process text-based information as quickly and easily as relational data. Oracle context 
analyses the contents and understands the structure of the English text it reads. The Oracle Release 7.3 ConText option consists of two separate, yet closely interrelated functions: a text management architecture contained entirely within Oracle7 and a text retrieval feature which uses natural language processing technology to identify themes and content in text. It is also capable of analysing the thematic content stored text and generating automatic summaries.

By breaking down the text into its constituent grammatical elements and determining how these elements contribute to the overall meaning, ConText works to understand the text it processes. It then uses this knowledge to produce a database index which can identify the development of key themes and determines their relative prominence [40]. Unlike other products that simply count words or use a hierarchical thesaurus to determine the main theme of a document, ConText parses every sentence in a document to determine the relative weight of the different themes. The Oracle ConText Lexicon is the heart of this text retrieval system. The Oracle ConText Lexicon contains a vast dictionary of over 1,000,000 words and phrases as well as the linguistic rules that bind them into thematic units. The lexicon is designed to recognise the vocabulary used in over 1,000 industries and can be augmented by user dictionaries. The ConText option provides automatic text reduction, which creates summaries conveying the main ideas and concepts of full documents. In addition to text reduction, ConText contains a powerful text classification feature which categorises documents according to linguistically identified themes rather than word frequency and statistics.

\subsubsection{Nordic WAIS/World Wide Web Project}

The Nordic WAIS/World Wide Web Project works on Improving Resource Discovery and Retrieval on the Internet [3]. The Nordic's automatic classification depends on UDC [3], an English medium classification scheme. The dynamic nature of the information sources on the network makes it necessary to have automatic tools that index and classify material. The algorithm used is as follows:

1. From the different fields of the selected document, words are extracted into a number of groups:

- words from the description field

- words from the subject field

- words from the keyword-list field

- words from the description field marked as keywords together with the name of the database.

2. A list of suggested classifications is constructed by comparing words from these groups with UDC's vocabulary. When a match between the vocabulary and a word is found the corresponding classification is added (restricted to the top 2 levels) to the list of suggested classifications with a certain weight. The weight depends on which group the matching word originates from. As an illustration, keywords in the subject field have higher weights than ordinary words in the description field. 
3. From the list of suggested classifications, the final classification is made. It is based on the accumulated weights for each proposed classification.

The Nordic project is not tied to UDC but can be used with other classification schemes such as the Library of Congress in order to produce different views of the subject trees.

\subsubsection{Harvest's Essence}

Essence's main objective is to extract indexing information from an input document. Content indexing requires type-specific processing to extract information effectively. By exploiting the semantics of common file types ${ }^{1}$. Essence generates compact yet representative file summaries that can be used to improve both browsing and indexing in resource discovery systems [22]. Essence decomposes the information extraction problem into four components that are independent of how data are stored, updated or exported. The components are listed below:

1. The type recognition step that uses various methods to determine a file's type. Essence recognises file types using a combination of file and site naming conventions, content testing, and user defined methods. The two main type recognition steps are:

- Naming conventions and heuristics.

- Examining file contents in determining the file types.

2. The presentation unnesting step that transforms nested files into an unnested format. When a presentation nested file is encountered, it is unnested into one or more result files. The result files themselves can also be nested. In addition to unnesting the input files, the presentation unnesting step also keeps a record of the nested origin of each unnested file, for use by the candidate selection and summarising steps.

3. Candidate selection step, selects which objects are to be summarised. Given a set of typed objects, the candidate selection step chooses objects to summarise. It attempts to eliminate redundancy among related files.

4. The summarising step, which applies a type specific extraction procedure to each selected object.

\subsection{Conclusions}

In this chapter, we described approaches in information retrieval, document indexing and text classification. We will be using some of these ideas in our system. Since the term position in the document is weighted, we will give an importance of the term location in the document. Since some of the available experimental evidence indicates that the use of abstracts in addition to titles brings substantial advantages in retrieval effectiveness [50], and since Salton's SMART system reveals that using the abstract rather than the whole

\footnotetext{
${ }^{1}$ See The Summariser's functions for each document table at the end of this subsection
} 
text gives the best results in information retrieval, the abstract and the title are used as two of the components of the Semantic Header. Since the titles could be good candidates for document specific concepts as Simone et al stressed, we will assign high weights to the terms located in the abstract and title fields. The additional utilisation of full texts of the documents appears to produce very little improvement over titles and abstracts alone in most subject areas [50]. In addition to assigning term weights, our system used the term frequency of occurrence addressed by Luhn.

The automatic classification approach used in ACTION relates the significant keywords to a set of available classes. Our system's thesaurus concept will be based on this idea; however, our system relates controlled terms with a set of subject headings. Our document classification scheme is based on Nordic's classification scheme. Nordic classifies documents by looking for a match between a set of vocabulary and the words in the document. Nordic uses words extracted from a set of groups and UDC's vocabulary to classify a document. Each classification gets a weight depending on which group the matching words originated from. The classification having the highest weight is selected. Our system will look for a match between a set of different weighted terms generated from the document and a set of controlled terms. The highest weighted subject headings associated with the matched controlled terms will be selected.

Luhn's automatic abstracting idea will be used in generating an abstract for a document and Harvest's file type recognition will be implemented in our thesis.

\section{ASHG's Thesaurus}

\subsection{The Thesaurus in IR}

A thesaurus is a set of items (phrases or words) plus a set of relations between these items [25]. The Thesauri commonly used in IR have shown inconsistent effects on retrieval effectiveness, and there is a lack of viable approaches for building a thesaurus automatically [25]. There are two types of manual thesauri. The general purpose and word based thesauri like Roget's and WordNet contain sense relations such as antonym and synonym but are rarely used in IR systems. The IR oriented and phrase based thesauri such as INSPEC, Library of Congress Subject Headings (LCSH), and Medical Subject Headings (MeSH) are widely used in commercial systems [25]. The major drawback of both types are that they are expensive to build and hard to update in a timely manner.

This paper addresses the issue of constructing a thesaurus in a semi-automatic fashion.

We used a number of rules in merging the subject headings found in INSPEC [62], LCSH [66] and ACM [67]. 


\subsection{ASHG's Thesaurus}

The ASHG's Thesaurus is composed of a three level subject hierarchies and a set of control terms associated with the subject headings found in the subject hierarchies. The Thesaurus used by ASHG contains four object classes: Level_0 which represents the general subject of the subject hierarchy, Level_1 which represents the sub-subject of the general subject and is derived from Level_0; Level_2 which represents the sub-subject of the Level_1 subject and is derived from Level_1. and finally Control_term which contains the root terms that are derived from the subject headings. A root term is the origin of all possible terms that can be generated from it by adding the suffixes and prefixes.

\subsubsection{The Subject Hierarchies}

Since different subject headings may be used to convey the same subject, and since different people may have different perspectives on the same single subject, controlled subject headings were derived. The CINDI system focuses on the standardisation of subject headings. This database helps the provider of the primary resource in selecting the correct subjects and sub-subjects' headings for the semantic header entry. CINDI's subject hierarchy is made up of three levels, where level_o contains the general subject heading. Currently we have included only two general subject headings: Computer Science and Electrical Engineering. Level_1 contains all the subjects that fall under level_o subjects, and similarly level_2 will contain more precise subjects that fall under level_1 subjects.

\subsubsection{Building CINDI's Classification}

ACM, INSPEC and LCSH were the main building blocks of CINDI's three level Subject Hierarchy. ASHG's computer science subject hierarchy used ACM's subject hierarchy as the starting point, and ASHG's electrical engineering subject hierarchy was based on INSPEC's subject hierarchy. We have exploited LCSH's subject headings relations to refine both hierarchies. LCSH contained relations between subject headings such as BT (Broader Term), NT (Narrow Term), UF (Used For), and RT (Related To). In order to augment ACM and INSPEC subject hierarchies, a search for an ACM or INSPEC subject heading was made in LCSH. If a match was found, the narrow terms found in LCSH under the matched subject were added to the list of subjects or terms under the ACM or INSPEC's matched subject heading.

This augmentation produced a hierarchy composed of five or six levels. Since CINDI's subject hierarchy was limited to only three levels, rules were applied to merge these subject headings. The resulting subject hierarchy was formed of three level subject hierarchy and one additional level. This last level contains terms used as control terms associated with the Level_2 subject headings. Merging the subjects of different levels involves the following rules:

1. The Computer Science's subject hierarchy's general (Level_O) subject is Computer Science. The Electrical Engineering's subject hierarchy's general (Level_O) subject is Elec- 
trical Engineering. Similarly, other subject hierarchies will be disciplined based as in the LCSH.

2. Level_1 and Level_2 subject headings found in the augmented ACM (or INSPEC) were merged to form one level, the CINDI's Level_1 subject heading. For some of the subject headings found in Level_2 which contained the subject headings found in Level_1, the Level_1 subject headings were dropped. The same rule was applied on subject headings found in Level_3 and Level_4 to give the CINDI's Level_2 subject heading. For example, Software and Software Engineering were found in the augmented ACM Level_1 and Level_D subject headings respectively. We dropped Software, to yield Software Engineering as CINDI's Level_1 subject heading.

3. Some of the subject headings found in the Level_1 and Level_D augmented ACM (or INSPEC) subject hierarchies were concatenated with a colon to form the CINDI's Level_1 subject heading. The same rule was applied on subject headings found in the augmented ACM (or INSPEC) Level_3 and Level_4 to yield CINDI's Level_2 subject heading. For instance, Office Automation and Spreadsheets were found in the augmented ACM (or INSPEC) Level_3 and Level_4 subject headings respectively. We concatenated them to derive CINDI's Level_2 subject heading, Office Automation: Spreadsheets.

4. The Level_5 and Level_6 augmented ACM (or INSPEC's) subjects were used as controlled terms associated with CINDI's Level_2 subject headings. Copyrights, for example, was used as a control term associated with CINDI's Level_d subject heading, Hardware and Software Protection

\subsubsection{The Control Term Subject Association}

The CINDI system uses a thesaurus to help the user in the registering and search processes. One such need for a thesaurus is in avoiding chaos introduced by differences in perception of different indexer. Hence, some form of standardisation of terms used has to be enforced. The main reason behind the Control Term Subject association is to extract or classify the primary source under a number of subject headings by comparing the significant list of words contained in the document with the list of controlled terms. An association between the controlled terms and their corresponding subject headings is created.

Each controlled term has three lists of subject headings attached to it. The three lists correspond to the general subject headings, sub-subject Level_1 subject headings, and Level_2 subject headings. Our controlled terms were based on the terms found in CINDI's subject hierarchy and the additional terms that are associated with CINDI's Level_2 subject headings. For each subject heading found in CINDI's subject hierarchy and the additional terms, we used their constituent English none noise words as their corresponding controlled terms. For example, the control term compute will be associated with Computer Science general subject heading. Similarly, the control term hardware will be associated with Hardware integrated circuits and Hardware performance and reliability level_1 subject headings and Hardware Simulation Design Aids level_2 subject heading. Each controlled term is associated with one or more subject headings. 


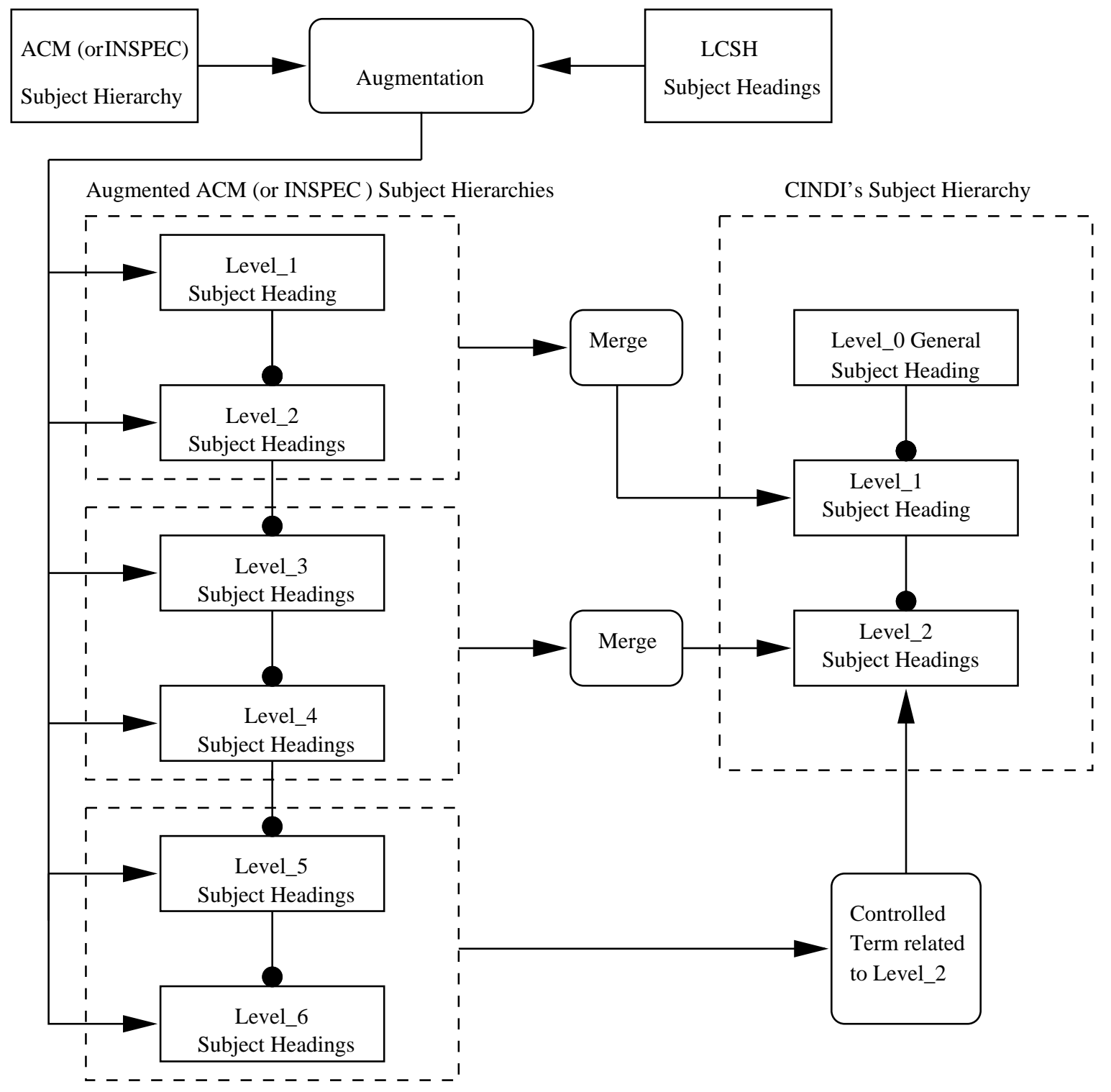

Figure 1: Transforming ACM (or INSPEC) Subject Hierarchy into CINDI's Subject Hierarchy 
Building the Controlled Terms: The subject headings found in CINDI's Level_0, Level_1 and Level_2 will be used as the basis for finding the controlled terms. In addition, the additional terms associated with CINDI's Level_2 subject headings are mapped into some controlled terms. Mapping CINDI's subject headings terms into controlled terms involves:

1. Since the controlled term dictionary is only composed of significant words, English stop words are removed from CINDI's subject hierarchy headings and the additional terms associated with CINDI's Level_2 subject headings. English Stop words are found in Table 2.

2. Applying ASHG's stemming process to the remaining list of words in order to get their root, which will be stored in the list of controlled terms.

3. Generating a list of words to be added to the spell check dictionary. These words are found in the subject headings but not in the spell check dictionary. Words like $W W W$ would be checked as wrong by the Unix spell check command, because $W W W$ is not found in the spell's dictionary. So, $W W W$ should be an added to the list of controlled terms.

Associating the controlled terms with the subject headings A document often covers a number of subjects or domains. Naturally some of them are of higher importance than others. CINDI uses the words in a document to classify it under a list of subject headings. This list of words from the document are matched against the controlled terms, generated above. The association between the subject headings and the controlled terms is constructed by comparing the root words found in these subject headings with the CINDI's controlled terms. If a match is found, then this subject heading is associated with the controlled term. The reason behind building such an association is that ASHG will generate a suggested list of subject headings using the words found in the document by consulting the Controlled term subject association. A summary of the steps used is discussed below:

1. Split each subject heading and the terms associated with CINDI's Level_2 subject headings into the words they are made up of.

2. English noise words found in the list of words are removed.

3. Words are checked using the spell command.

4. Similarly, words not found in the spell Unix dictionary and the new added words are dropped.

5. Apply the stemming process to generate the root controlled terms from the words.

6. Each root controlled term will be associated with the subject headings that contains it.

For example, Theory of computation by abstract devices subject heading is divided into the following words: theory, of, computation, abstract, by, and devices. The English noise words such as of and by are dropped. Steps 3, 4 and 5 are applied on the remaining terms. The generated root terms such as abstract, theory, computation, and device are associated with the initial subject heading. 


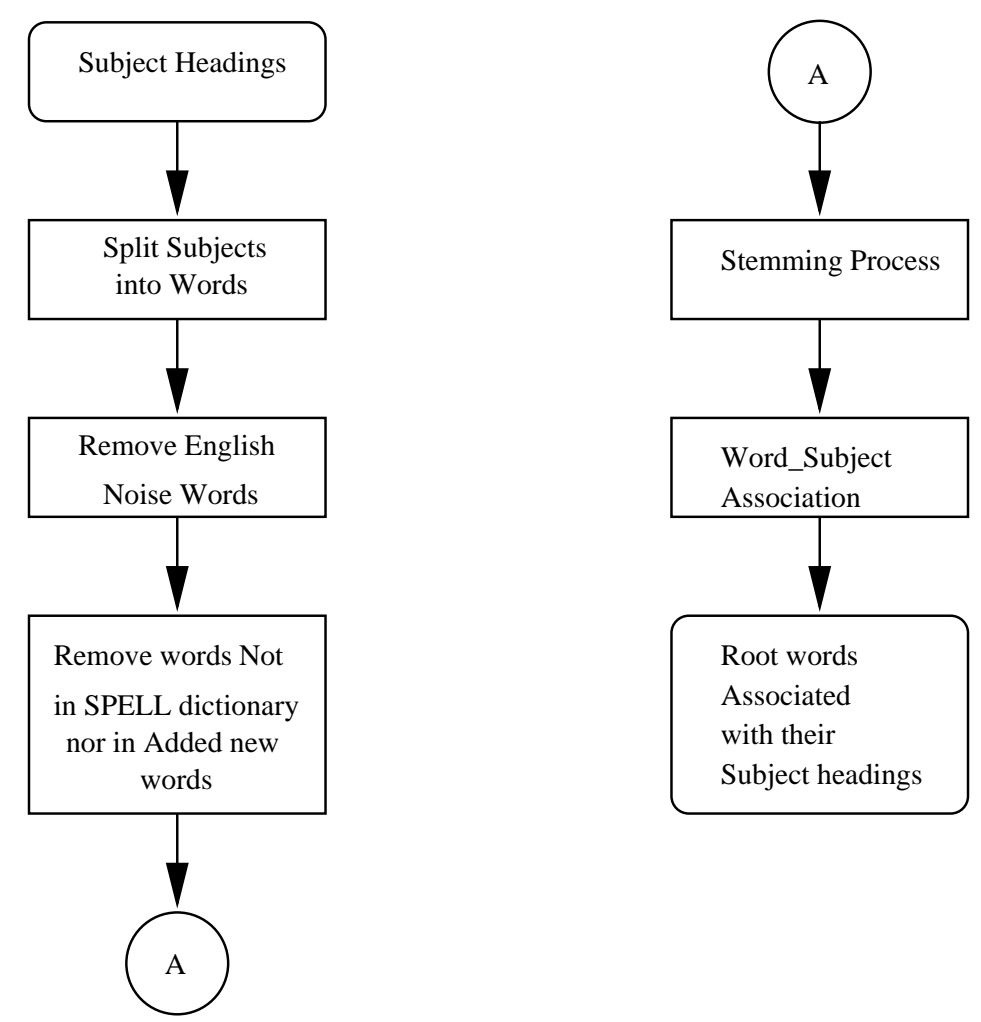

Figure 2: Associating words' roots to their subject headings 


\begin{tabular}{|c|c|c|c|c|c|c|}
\hline $\mathrm{I}$ & you & she & he & it & we & mine \\
\hline they & me & her & him & us & them & yours \\
\hline hers & his & its & ours & theirs & their & my \\
\hline this & that & the & these & those & who & whom \\
\hline which & what & whoever & whomever & whichever & whatever & all \\
\hline any & anybody & anyone & anything & each & everybody & everyone \\
\hline everything & few & many & nobody & none & one & several \\
\hline some & somebody & someone & myself & yourself & herself & himself \\
\hline itself & ourselves & $\mathrm{a}$ & more & less & also & consequently \\
\hline finally & furthermore & hence & however & incidentally & indeed & instead \\
\hline likewise & meanwhile & nevertheless & next & nonetheless & otherwise & still \\
\hline then & therefore & thus & forever & moreover & only & are \\
\hline is & afterwards & again & almost & alone & already & always \\
\hline about & above & across & after & against & along & among \\
\hline around & at & before & behind & below & beneath & beside \\
\hline between & beyond & but & by & despite & down & during \\
\hline except & for & from & in & inside & into & like \\
\hline near & of & off & on & onto & out & outside \\
\hline over & past & since & through & throughout & till & to \\
\hline toward & under & underneath & until & up & upon & with \\
\hline within & without & amongst & anyhow & anything & anywhere & be \\
\hline became & become & becomes & becoming & been & beforhand & being \\
\hline besides & can & and & but & or & nor & for \\
\hline so & yet & after & although & as & because & before \\
\hline how & if & once & since & than & that & till \\
\hline though & until & when & where & whether & while & both \\
\hline either & neither & whether & an & another & & \\
\hline
\end{tabular}

Table 1: Noise (Stop) words extracted by ASHG

\section{ASHG}

\subsection{Introduction}

In this section, we present the Automatic Semantic Header Generator (ASHG) of the CINDI system. This is an important step in building the Semantic Header. To save time for the document's provider, ASHG provides an initial set of subject classification and a number 
of components of the Semantic Header for the document. The design goal of ASHG is to automatically build a reliable Semantic Header, which includes classifying a document under a list of subject headings. ASHG's scheme is measuring both the occurrence frequency and positional weight of keywords found in the document. Based on the selected document's keywords, ASHG assigns a list of subject headings by matching those keywords with the controlled terms found in the controlled term subject association.

The ASHG extracts some of the meta-information from a document and stores it in a Semantic Header. For example, when a new document is presented to the system, fields such as document's title, abstract, keywords, dates, author, author's information, size and type are extracted. Using frequency occurrence and positional schemes, ASHG measures the significance of the words found in the previously mentioned list. Word stemming is used in order generate a base form for each word. The system tries to match the base forms of the words with the controlled terms found in the controlled term subject association. If a match was found the subject headings associated with the controlled terms are extracted and ranked accordingly. The major steps followed by ASHG are briefly described below:

1. Document Type Recognition: In order to apply the correct ASHG to a document, the type of the document has to be recognised. The system currently understands HyperText Markup Language (HTML), Latex and plain text documents.

2. Applying ASHG's Extractor: The summariser corresponding to the type of document is applied to the input document.

3. ASHG's Document Classification: The document is assigned subject headings. It involves:

(a) Word stemming: The system applies the stemming process ${ }^{2}$, to map the words found in the extracted fields onto a base root word.

(b) A Look up into the Controlled Term Subject dictionary.

4. Semantic Header Validation: The generated Semantic Header is presented to the user to validate.

\subsection{Document Type Recognition}

When a document is submitted to the system, the system tries to recognise the type of the document through the name conventions. If it fails the system will then examine its contents. If a failure arises following the examination of the content, the system informs the user that the document type is unrecognised, and the user is asked to enter the Semantic Header. Naming conventions and heuristics and Examining file contents are the two steps used in the document type recognition process.

\footnotetext{
${ }^{2}$ The stemming process will be discussed in more details in section 5.3.5
} 
The document file upon submitting is passed to a function, which checks the document's name extension. If the naming conventions fails in recognising the document type, the system examines the file content. The semantics of the ASHG's types are exploited when attempting to recognise the file type. Finally, the user either confirms or rejects the result. If the user rejects the result, he should choose a type from a list that is displayed. If the user confirms the document's type as recognised, ASHG applies the extractor corresponding to the type confirmed by the user. Otherwise, he should choose a type and then apply ASHG. If the file type is unrecognised by $\mathrm{ASHG}$, the system extracts the size of the file and the date of creation.

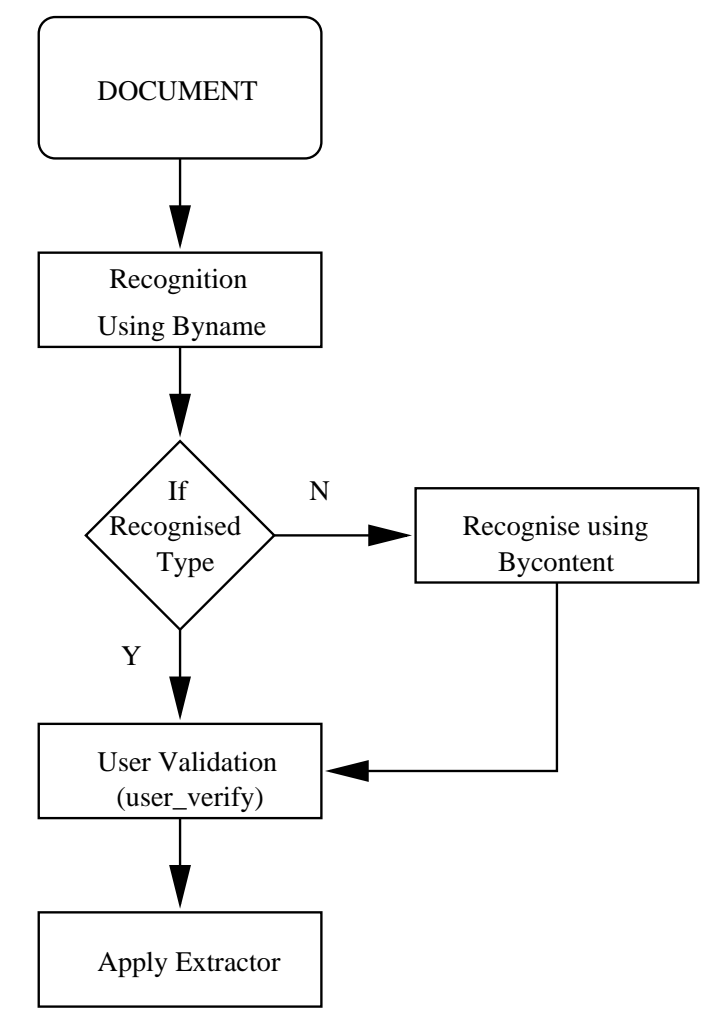

Figure 3: Document Type Recognition

\subsection{Applying ASHG's Extractors}

Based on the document's type uncovered in the document type recognition step, ASHG applies an extraction procedure. ASHG uses its understanding of HTML, Latex and text syntax documents to extract the document's meta-information. ASHG's HTML_extractor, LATEX_extractor, TEXT_extractor and UNKNOWN_extractor are applied to HTML type documents, to Latex type documents, to Text type documents and to unrecognised type document respectively. 
Using the document's syntax, ASHG extracts summary information, such as the title, keywords, dates of creation, author, author's information, abstract and size. In both HTML and Latex documents, the author might explicitly tag some of the fields to be extracted. In case these fields were not explicitly tagged, ASHG attempts to extract them using some heuristics. For example, extracting the keywords in an HTML document, The HTML_extractor extracts words that are found in the meta tag field, if they were included by the author. However, if the explicit keywords were not found in the document, then words found in the title, abstract and other tagged words would be used to extract an implicit list of keywords.

\subsubsection{HTML_extractor}

HTML is designed to specify the logical organisation of a document, with important hypertext extensions [21]. An HTML document is designed in a way to mark selections of text as titles or paragraphs, and then leaves the interpretation of these marked elements up to the browser. The HTML_extractor exploits this mark-up in order to extract the metainformation.

ASHG exploits both the HEAD's TITLE mark-up element and the HEAD's META markup element, which is a general element for document meta-information. The META mark-up element contains information such as date of creation, and date of expiry. It can also contain Arbitrary User-Specified Information, which includes information such as keywords, name of the author, and a summary of the document. In case these mark-up elements are not found in the HTML document. ASHG extracts the meta-information by applying some heuristics that will exploit the $B O D Y$ mark-up elements such as the $H n$ headings, $P$ paragraphs, $A D D R E S S$ Address, Blockquote, Lists and text emphasis.

HTML_extractor extracts the title, explicitly stated keywords, language (English), author(s), dates (Created, Expiry), size of the file, and the abstract from an HTML document. Generating an implicit list of keywords will be discussed in sub-section 5.3.5, and the subject headings classification scheme is described in section 5.4. Both procedures are standard for all extractors.

1. Extracting the author from an HTML document: The HTML_extractor extracts the author from the META mark-up element. For instance, if the HTML document contains <META name = "author" content $=$ "Sami Haddad" >, the HTML_extractor extracts Sami Haddad as the author of the document.

2. Extracting dates from an HTML document: Document's creation and expiry dates could be found in the META mark-up element, for example <META name $=$ "Created" content $=$ "18/03/98" >. The HTML_extractor extracts both the date of creation and date of expiry. If it fails to locate them in the META mark-up element, it uses the stat and GM-time commands to extract the date of creation. stat unix command contains information about the file such as File size in bytes, Time of last access, Time of last data modification and Time of last file status change. GM-time unix command converts the time to Coordinated Universal Time (UTC), which is what the UNIX system uses internally. 


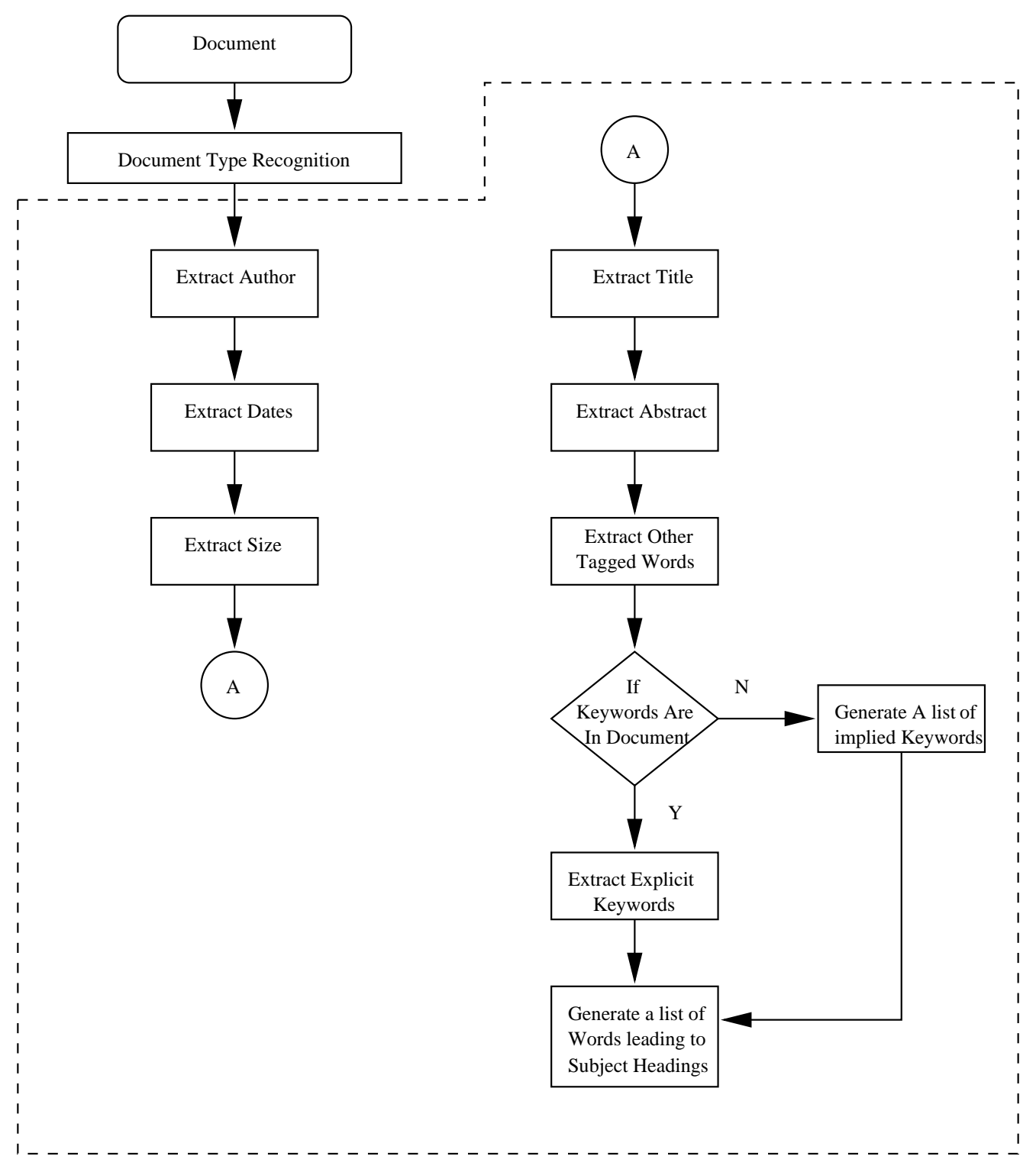

Figure 4: ASHG's extraction steps 
3. Extracting the size of the HTML document: Using the stat unix command, the size of the file can be extracted.

4. Extracting the title from an HTML document: The title, which is found in between <title $>$ and $</$ title $>$ tags, is extracted. For example, if the HTML document contains < title $>$ Cindi System analysis </title $>$, HTML_extractor extracts Cindi System analysis as the document's title. If the title tags were not found in the $H T M L$ document, then the HTML_extractor will extract the first heading found in between $<\mathrm{h} 1>$ and $</ \mathrm{h} 1>$ tags. If it fails, then the first sentence is extracted after generating an HTML tag-free document.

5. Extracting the abstract from an HTML document: The HTML_extractor attempts first to extract the content from the META abstract mark-up element. If it fails to find the abstract in the META mark-up element, it extracts the paragraph headed by the tagged word abstract. If it fails to locate an abstract heading, it applies an automatic abstracting method. This method, which is similar to Luhn's automatic abstracting method described in chapter 3, attempts to extract a section or paragraph that is headed by introduction. Based on the number of significant root words in the sentence, a numerical measure is developed for a sentence. The automatic abstracting includes the highest measured sentences in the abstract. If it fails, the HTML_extractor extracts the first paragraph after removing the HTML tags and applies the automatic abstracting method, described above, on this paragraph.

6. Extracting other tagged words from an HTML document: The HTML_extractor extracts a list of tagged words. For example, if the HTML document contains the meta tags $\langle\mathrm{b}\rangle$ Database $\langle/ \mathrm{b}\rangle$, the HTML_extractor includes Database in the list of other tagged words. This list of words is used in generating an implied list of keywords and in generating a list of significant words used in the document classification scheme. Both processes will be described in subsection: 5.3.5.

7. Extracting explicitly stated keywords from an HTML document: The HTML_extractor attempts first to extract the keywords from the META mark-up element. If it fails, it extracts the list of keywords following the tagged word, keywords. For example, if the HTML document contains the meta tags $\langle\mathrm{b}\rangle$ Keywords $\langle/ \mathrm{b}\rangle$ : Bibliographic record, search engineering, analysing search, Content description, Database Systems, Expert System, Indexing applications, Searching, $U R C<\mathrm{p}>$, the HTML_extractor extracts these as the document's keywords.

\subsubsection{Latex_extractor}

LaTeX is a TeX macro package, originally written by Leslie Lamport [31], that provides an easy way to use the TeX document processing system. LaTeX allows mark-up to describe the structure of a document, so that the user need not think about presentation. Latex commands describe the structure of the document. There is a list of things that should be realized about these commands:

1. All Latex commands consist of a backslash followed by one or more characters. They should be typed using the correct mixture of upper and lower case letters. 
2. Some commands are placed within the text. These are used to switch on and off things, like different type styles.

3. There are other commands that look like \command $\{$ text $\}$

4. When a command's name is made up entirely of letters, its end is marked by something that isn't a letter. The mark, for instance, could be a space.

The LATEX_extractor exploits the use of mark-up elements of specifically the Latex article style to extract the meta-information. It extracts the title, explicitly stated keywords, language (English), author(s), dates (Created, Expiry), size of the file, and the abstract from a Latex document. Generating both implicit keywords and a list of subject headings for a document will be described in a later section, since they are a standard procedure for all extractors.

1. Extracting the author from a Latex document: The \author $\{$ names $\}$ command declares the author(s), where the name(s) is a list of authors separated by $\backslash$ and commands. The $\backslash \backslash$ is used to seperate lines within a single author's entry. For example, to give the author's institution or address. If the following was in the latex document:

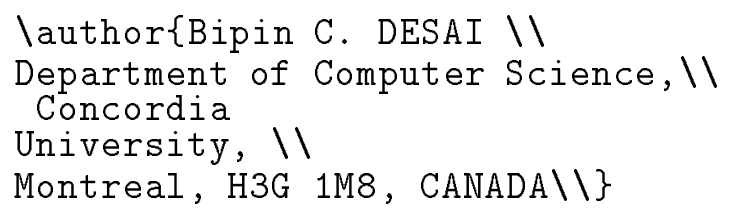

LATEX_extractor extracts Bipin C. DESAI as the author's name and Department of Computer Science, Concordia University, Montreal, IISG 1M8, CANADA as the author's address.

2. Extracting dates from a Latex document: The \date\{text $\}$ declares text to be the document's date. For example, if " $\backslash$ date $\{18 / 04 / 98\}$ " was found in the Latex document, LATEX_extractor extracts $18 / 04 / 98$ as the document's date. If no \date command is found in the Latex document, LATEX_extractor uses the stat and GM-time commands to extract the date of creation.

3. Extracting the size of the Latex document: Using the stat unix command, the size of the file can be extracted.

4. Extracting the title from a Latex document: The \title\{text $\}$ command declares text to be the title. The LATEX_extractor exploits the title mark-up element to extract the title. For instance, if the latex document contains \title\{CINDI system analysis \}, LATEX_extractor extracts CINDI system analysis as the title. If it fails, $L A T E X_{-}$extractor exploits the following mark-up element:

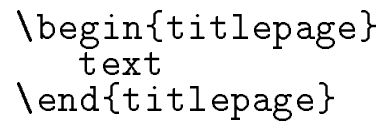


It extracts the first sentence found in the text. If it fails, it extracts the text marked up by huge, or large. It can exploit the presence of \begin } \{ \text { huge } \} \text { text } \backslash \text { end } \{ \text { huge } \} \text { or } $\backslash$ huge $\{$ text $\}$. If it fails, it exploits the presence of \begin } \{ \text { Large } \} \text { text } \backslash \text { end } \{ \text { Large } \} \text { or } $\backslash$ Large $\{t e x t\}$. If none of the above mark-up elements were found in the document, $L A T E X_{-}$extractor filters out all latex mark-up elements and extracts the first sentence as the document's title.

5. Extracting the abstract from Latex document: A latex document might contain $\backslash$ begin $\{a b s t r a c t\}$ text $\backslash$ end $\{$ abstract $\}$. If it does, the LATEX_extractor extracts the text as the document's abstract. Otherwise, it extracts the sections which are headed by the word abstract. For example, if \section $\{$ Abstract $\}$ is found in the document, the paragraph that follows is extracted. However, if it fails, it extracts the paragraph that follows \huge $\{$ Abstract $\}$, \large $\{$ Abstract $\}, \backslash$ bf $\{$ Abstract $\}$, or $\backslash$ it $\{$ Abstract $\}$. If none of these are found, the automatic abstracting method is applied. This method, which is similar to Luhn's automatic abstracting method, is described in chapter 3 and in the HTML_extractor. If the automatic abstracting method fails, the first marked up paragraph is extracted, otherwise, all the latex mark-ups are removed and the first paragraph is extracted as the document's abstract.

6. Extracting other tagged words from a latex document: The LATEX_extractor extracts a list of other marked up words. It uses the sectioning commands and the three typefaces latex commands: \em (Emphatic), \bf (Boldface) and \it (Italic) to extract the marked up words. The extracted words will be used in the generation of an implicit list of keywords and the generation of a list of significant words used in the document's classification scheme. This process of generating an implicit list of keywords and a list of significant words is described in subsection 5.3.5. Here are the Latex sectioning commands: \part, \chapter, \section, \subsection, \subsubsection, $\backslash$ paragraph, and \subparagraph.

7. Extracting explicitly stated keywords from a latex document: LATEX_extractor exploits three typefaces latex commands: \em (Emphatic), \bf (Boldface) and \it (Italic). These commands are used inside a pair of braces to limit the amount of text that they affect. For instance, if the following was in the latex document: $\{\backslash$ bf Keywords: $\}$ Information retrieval, Modelling, meta-data, cataloguing. searching, discovery, information resources, $W W W$, Internet, resource discovery $\backslash \backslash$ , the LATEX_extractor extracts the words as the document's keywords.

\subsubsection{Text_extractor}

Perhaps one of the most challenging tasks in information extraction is to extract and manipulate information found in plain text documents. Since these documents do not contain tags or mark-up elements, the TEXT_extractor relies heavily on heuristics in extracting the title, explicitly stated keywords, language (English), author(s), dates (Created, Expiry), size of the file, and the abstract from a Latex document. Generating both implicit keywords and a list of subject headings for a document will be described in a later section.

1. Extracting the author from a plain text document: The TEXT_extractor looks for a pattern such as written by, edited by or revised by. If it finds one of them, it extracts the text following it and stores it as the author's Semantic Header field. 
2. Extracting dates from a plain text document: The TEXT_extractor uses the stat and GM-time commands on the document file to extract the date of creation.

3. Extracting the size of the plain text document: Using the stat unix command, the size of the file can be extracted.

4. Extracting the title from a plain text document: When presented with a plain text document, the TEXT_extractor extracts the first sentence from the document. This sentence is used as the document's title. If it fails, it generates a list of sentences by extracting all sentences found in the first, second and last paragraph and by extracting the first sentence of all other paragraphs. Each sentence is divided into its constituent words. After dropping all English Noise or Stop words, the remaining words are stemmed. Each sentence is given a weight according to the frequencies occurrences' sum of the stemmed words found in the sentence. The TEXT_extractor selects the highest weighted sentence as the document's title.

5. Extracting the abstract from plain text document: The TEXT_extractor looks for the pattern, abstract, and extracts the first paragraph following it. If it fails, the automatic abstracting method is applied on the document's introduction. If it fails to construct an abstract, TEXT_extractor applies the automatic abstracting method on the sentences found in the first, second and last paragraph and on the first sentence of all other paragraphs. The sentences are divided into their constituent words. Dropping all English Noise words, the remaining words are stemmed. The extracted sentences are weighted according to the frequency occurrence of the stemmed words. The TEXT_extractor will construct the document's abstract by extracting the highest weighted sentences.

6. Extracting other words from a plain text document: The TEXT_extractor extracts the words found in the first two paragraphs, the last paragraph and in the first sentence of each other paragraph. After removing the English Noise words, a list of stemmed words is derived. The derived words will be used in the generation of an implicit list of keywords and the generation of a list of significant words used in the document's classification scheme.

7. Extracting explicitly stated keywords from a plain text document: The TEXT_extractor extracts the text following the word keyword as the document's keywords, until the TEXT_extractor reaches an introduction heading or a new paragraph.

\subsubsection{Unknown_extractor}

ASHG supports HTML, Latex and Text documents; however, if the document is not any of these types, ASHG applies the UNKNOWN_extractor. It extracts the size of the document and the creation date. It is up to the document's author or provider to enter the Semantic Header's information.

\subsubsection{Generating an implicit list of keywords and words used in Document classification}

ASHG generates an implicit list of keywords in case explicit keywords were not found in the document. It derives a list of most significant words, which is used in the document 
classification scheme. In case keywords were not found in the document, the system derives a list of words from the words found in the title, abstract, and other tagged fields. This list of derived words will also be used in classifying the document. However, if the keywords were explicitly stated in the document, then ASHG will generate a list of words from the words found in the title, abstract, keywords and other tagged fields. This list is used to assign a list of subject headings for the document.

Generating both lists of words relies on the stemming process that will map the words into their root words, the stemmed word frequency of occurrence and the word location in the document. It uses the following algorithm in generating the list of implicit keywords, in case the keywords were not found in the document, and the words used in the classification scheme:-

1. Extract the title, abstract and other tagged fields. If the document wasn't tagged such as in a plain text document, words found in the first two and last paragraphs and in the first sentence of each paragraph are selected. Keywords are extracted if they were found in the document.

2. English Noise words constitute usually around 30 to 50 per-cent of a document. The Information Retrieval community calls them the Stop List. These words are dropped from the extracted fields.

3. The remaining words are sent to the stemming process. This process will remove the words' suffixes and prefixes. For example, the words: cycled, cycler, cycling and cycles are stemmed to the root term, cycle. The aim of the stemming process is to generate base word class, which include all the forms that could be generated from it.

4. Because the terms are not equally useful for content representation, it is important to introduce a term weighting system that assigns high weights for important terms and low weight for the less important terms [55]. Therefore, the weights constitute the importance of a word. The system assigns weights to both lists of root words. The weight assignment uses the following scheme:

(a) If the word appears in the explicitly stated keywords, it is assigned a weight of five 3 . Since authors explicitly state the keywords to convey some important terms, which their document covers, it is assigned the highest weight. For example, if the word device is found in the list of explicitly stated keywords, the word device is assigned a weight of five.

(b) Usually, words found in the abstract are the second most important words, because this is where the author tries to convey his/her idea. Therefore, words found in the abstract are the second most significant and they convey the idea of the article more than any words found in other locations [51]. If the word appears in the abstract, it is assigned a weight of four.

(c) If the word appears in the title, it is assigned a weight of three. For example, if the word compute is found in the title, it is assigned a weight of three.

\footnotetext{
${ }^{3}$ If the keywords are stated, then they will be used in addition to the other weight classes in determining the subject classification for the document
} 
(d) If the word appears in the other tagged words, it is assigned a weight of two.

5. Each numeric weight is a class by itself defining the words' location. The system has the following classes:

(a) A class weight of two defines the OTHER WORDS class. This class contains the terms found in only the OTHER WORDS field.

(b) A class weight of three defines the TITLE class. The class three contains all the terms found only in the title field.

(c) The class weight four contains all the terms found only in the abstract field. Therefore a class weight of four defines the ABSTRACT class.

(d) A class weight of five includes all the terms found in either the keywords' field or in the title and other words fields.

(e) A class weight of six includes all the terms found in both the abstract field and the other words field.

(f) A class weight of seven includes all the terms found in either the keyword and other words fields or the abstract and title fields.

(g) A class weight of eight contains all the terms found in keyword and title fields. For example, if the word compute appears in both the title and explicitly stated keywords, it is assigned a weight of eight. The word compute will be an element of the class weight of eight.

(h) A class weight of nine contains all the terms found in either the abstract, title and other words fields, or abstract and keywords fields.

(i) A class weight of ten contains all the terms found in the other words, title and keywords fields.

(j) A class weight of eleven contains all the terms found in the other words, abstract and keywords fields.

(k) A class weight of twelve contains all the terms found in the title, abstract and keywords fields.

(1) A class weight of fourteen contains all the terms found in the other words, title, abstract and keywords fields.

A term appearing in other words field is less important than the one appearing in the abstract field. Furthermore, a term appearing in both title and other words fields is less significant than the one appearing in the keywords, abstract and title field. In a high class weight, we are interested in extracting more terms than in lower class weights. Thus, we tend to extract more terms from the high weighted classes. To limit the number of extracted terms, we use the term's frequency of occurrence. Significant terms have the highest frequency of occurrence in the low weighted classes. As the class weight increases, more of its terms are regarded as significant. To include more significant terms, the domain of the terms' frequencies is expanded. The more is the class weight, the wider is the domain frequency of the significant terms. 
For each class, we set the maximum class frequency to be the maximum frequency of occurrence of a term found in that class. For instance, if, in class four, we had three terms having two, four and six as frequencies, the system would select six as the maximum class four frequency. The words' frequencies are compared with their corresponding maximum class frequency. For low weighted classes such as two and three, significant terms have the maximum class frequencies. Thus, limiting the number of significant terms. However, all terms found in class eight and more are significant regardless of their frequency of occurrence.

\begin{tabular}{|l|l|}
\hline Term Weight & Term Frequency \\
\hline \hline 2 & Maximum Class 2 frequency \\
\hline 3 & Maximum Class 3 frequency \\
\hline 4 & Greater or equal to Maximum Class 4 frequency minus 1 \\
\hline 5 & Greater or equal to Maximum Class 5 frequency minus 1 \\
\hline 6 & Greater or equal to Maximum Class 6 frequency minus 2 \\
\hline 7 & Greater or equal to Maximum Class 7 frequency minus 3 \\
\hline 8 or more & All \\
\hline
\end{tabular}

Table 2: Weight and Frequency numbers used in extracting terms

6. Two lists of words will be generated. The first one containing only the root words or controlled terms found in CINDI's thesaurus. This list of controlled terms is used in the document's subject classification scheme. The second list contains the most significant root words not found in CINDI's thesaurus.

7. If no keywords were found in the document, ASHG extracts words having a term weight more than four and their corresponding frequencies of occurrence is the same as the ones tabulated. These words are the document's keywords.

8. In generating a list of controlled terms used to classify the document, terms having weight of two or more are extracted. The extracted words should have the frequencies of occurrence as the ones tabulated.

\subsubsection{ASHG's Stemming Process}

Stemming consists of processing a word so that only its stem or root form is left. Plural stemming attempts to identify and index the singular form of a term. Porter stemming attempts to identify and index the word stem. If a word and its stem are different, only the word stem is indexed. The stemming algorithm developed by Porter [44] at Cambridge uses weak stemming to remove common plural endings and other grammatical suffixes like -ing and -ed and implements strong stemming to remove derivational suffixes like -ent, -ence, and -ision. Many searchers use right hand truncation to find different variations of a search term that is of interest. The problem with right hand truncation is that it indiscriminately adds words to the query [72]. For example, if a searcher were to search for the truncated form of the word cover, the searcher would not only retrieve instances of the terms covers, 
covering and covered but also the terms covert, coverall, coversheet and coverage. QPAT-US [72] helps you avoid extraneous right hand truncation terms by automatically performing a process called stemming. First, QPAT-US evaluates your terms for common suffixes that indicate plurality, verb tense, etc. If QPAT-US discovers these suffixes, it will strip them to find the root form of the term. For instance, if QPAT-US finds the term covering it will strip the suffix to obtain the root word of cover. Next, QPAT-US takes the root form of your search terms and, using sophisticated linguistic rules, creates a set of word variants. If your original term is covering, QPAT-US will also search for cover, covers and covered.

ASHG's stemming process implements the removal of both suffixes and prefixes of a given word in order to get the root of the word. For example, applying the stemming process on the words simulation and analogies, the words simulate and analogy are generated as their root words respectively. ASHG stores the root forms of the words.

Suppose the word impressionists is in a document for which meta-information is to be extracted. Without stemming, this would match only the keyword impressionists and not the singular form. Now suppose that the word impressionist was in CINDI's list of controlled terms, then that document will miss that term and will not have it as a keyword. Following stemming, documents having the word impressionistic and impressionism will match the root term that is found in CINDI's list of controlled terms. We have mainly used the spell unix command in our system in extracting the root of a word. The spell command collects words from an input file and looks them up in a dictionary list. Words that neither occur among nor are derivable (by applying certain inflections, prefixes, and/or suffixes) from words in the spelling list are printed on the standard output. Two options were used along the spell unix command in our system: the $-v$ option, in which all words not literally in the spelling list are printed, and plausible derivations from the words in the spelling list are indicated, and the $-x$ option, in which every plausible stem is displayed, one per line, with = preceding each word. The steps of the ASHG stemming process are:

1. Using the sort unix command, sort the input words.

2. Apply the uniq unix command to filter out duplicate words.

3. Apply the spell command with the $-x$ option. Thus, all the plausible stems are stored in an output file.

4. Apply the spell command with the - v option. Thus, all words not found in the spelling list are stored.

5. Create a file which contains the words found in step 3 but not in step 4 .

6. Apply the spell command with the -v option to each word found in the file that resulted from the previous step. If the resulting output is empty, this means that this root word is found. 


\subsection{ASHG's Document Subject Headings Classification scheme}

An important step in constructing the semantic header is to automatically assign subject headings to the documents. The title, explicitly stated keywords, and abstract are not enough by themselves to convey the ideas or subjects of the document. Since the author tries to convey or to summarise his ideas in the previously mentioned fields, there is a need to use all English none noise words found in those fields. To assign the subject headings, ASHG uses the resulting list of significant words generated from the previous section and CINDI's controlled term subject association. The subject heading classification scheme relies on passing weights from the significant terms to their associated subjects, and selecting the highest weighted subject headings.

\subsubsection{The Algorithm followed}

Having the keywords, title words, abstract words and other tagged words, will help us select the most appropriate subjects for a given document. The following algorithm is used:-

1. Three lists of subject headings are to be constructed. The list of Level_0 subject headings, the list of Level_1 subject headings and the list of Level_2 subject headings.

2. For each term found in both CINDI's controlled terms and the generated list of words, the system traces the controlled term's attached list of subjects (list of levelo, level1 and level2) headings, and adds the subject headings to their corresponding list of possible subject headings.

3. Weights are also assigned to the subject hierarchies. The weight for a subject is given according to where the term matching its controlled term was found. A subject heading having a term or set of terms occuring in both title and abstract, for instance, gets a weight of seven. The matched terms' weights are passed to their subject headings.

4. The system extracts Level_2, Level_1 and Level_0 subject headings having the highest weights from the three lists of possible subject headings.

5. After building the three lists for the three level subject headings, the system :

(a) selects the subjects using the bottom-up scheme.

(b) Having selected the highest weighted level_2 subject headings, the system derives their level_1 parent subject headings.

(c) An intersection is made between the derived level_ 1 subject headings and the list of the highest weighted level_1 subject headings. The common level_ 1 subjects are the document's level_1 subject headings.

(d) The system uses the same procedure in selecting level_o subject headings. 


\subsection{Semantic Header Validation}

Once the process of extracting the meta-information is terminated, the semantic header is displayed for the source provider to modify, add or remove some of the attributes. Once the provider finishes, the semantic header can be stored in the CINDI database.

\section{Analysis of ASHG's Results}

In this section, we illustrate how the ASHG system extracts the meta-information from the HTML, Latex and text documents, and we demonstrate ASHG's automatic subject headings classification. For each of these document types, we apply ASHG and show the results. We compare the subject classification generated by ASHG with that of INSPEC for the same set of documents. We also compare the results with what the papers' authors would regard as good subject classifications and poor ones.

\subsection{Reduction of Controlled Terms}

Salton et al [55] introduces the term weighting system that assigns high weights to terms deemed important and lower weights to the less important terms. The term weighting system favours terms with high frequency in particular documents but with a low frequency overall in the collection.

ASHG's controlled terms favours the terms that have low frequency in the ASHG's subject headings over the terms having high frequency. Controlled terms having high frequency are dropped from the ASHG's list of controlled terms. Terms having lower frequency distinguish the subject headings associated for the document. The controlled term system occurs two hundred and eleven times in the ASHG's subject headings, which is the highest frequency control term. Therefore, it is dropped from the ASHG's list of controlled terms. Other control terms such as section, two, three, function, and method were dropped due to their ambiguity. The following table shows the words that are dropped and their corresponding frequencies.

\subsection{Experiments}

The experiments described here are designed to test the accuracy of the generated index and the subject headings classification results. After applying the ASHG on a set of documents, the generated index fields such as title, keywords, abstract and author are compared with those that are found in the document. The ASHG's automatic subject headings classification results are compared with the INSPEC's classification and with what the papers' authors would regard as good subject classifications and poor ones. 


\begin{tabular}{|l|l|}
\hline Words & Frequency \\
\hline \hline system & 211 \\
\hline power & 115 \\
\hline design & 106 \\
\hline electric & 100 \\
\hline circuit & 96 \\
\hline application & 93 \\
\hline language & 87 \\
\hline device & 84 \\
\hline measure & 83 \\
\hline general & 72 \\
\hline manage & 71 \\
\hline information & 70 \\
\hline analysis & 69 \\
\hline miscellaneous & 58 \\
\hline other & 47 \\
\hline
\end{tabular}

Table 3: Words Dropped from the list of controlled terms

The experiments were conducted on thirty three documents. The titles of these documents can be viewed in appendix A. These documents dealt with computer science and electrical engineering subjects. ASHG was able to extract all the explicitly stated fields such as title, abstract, keywords, and author's information with a hundred percent accuracy. If the abstract was not explicitly stated, ASHG was able to automatically generate an abstract that would describe the paper. However, ASHG's implicit keyword extraction generated a list of words which included some words that are insignificant. These insignificant words in turn lead to the diversion in subject classification.

We have consulted the papers' authors on the ASHG's subject classification results. Their response was divided into three categories: good, OK/Not sure and poor subject hierarchy selection. Good subject hierarchy selection implied that the authors would have chosen them as subject hierarchies for the documents. OK/Not sure subject hierarchy selection implied that the authors doubt the results and they would not choose them. Finally, the poor subject hierarchy selection implied that the selected subject hierarchies described another different subject. We compared the ASHG's subject classification results against the INSPEC's classification done by expert cataloguers and thesaurus. Some of the ASHG's subject classification had different words than INSPEC's even though they described the same subject. That was due to the fact that our computer science subject classification was built from ACM and not from INSPEC. 


\begin{tabular}{|c|c|c|c|c|c|c|}
\hline \multirow{3}{*}{$\begin{array}{c}\text { HTML } \\
\text { Document }\end{array}$} & \multirow{3}{*}{$\begin{array}{c}\text { Number of } \\
\text { Subject Headings } \\
\text { generated by ASHG }\end{array}$} & \multirow{2}{*}{\multicolumn{3}{|c|}{ Author's Opinion }} & \multirow{3}{*}{$\begin{array}{l}\text { Accuracy } \\
\text { A: Author } \\
\text { I: INSPEC }\end{array}$} & \multirow{3}{*}{$\begin{array}{c}\text { OK/Good's } \\
\text { Accuracy }\end{array}$} \\
\hline & & & & & & \\
\hline & & Good & OK/Not Sure & Poor & & \\
\hline D1 & 6 & 4 & 2 & 0 & $\begin{array}{c}66.66 \%(\mathrm{~A}) \\
16.6 \%(\mathrm{I})\end{array}$ & $100 \%$ \\
\hline$\overline{\mathrm{D} 2}$ & 7 & 4 & 1 & 2 & $57.14 \%(\mathrm{~A})$ & $71.42 \%$ \\
\hline D3 & 8 & 6 & 0 & 2 & $75 \%(\mathrm{~A})$ & $75 \%$ \\
\hline $\mathrm{D} 4$ & 9 & 3 & 4 & 2 & $33.33 \%(\mathrm{~A})$ & $77.77 \%$ \\
\hline D5 & 7 & 0 & 3 & 4 & $0(\mathrm{~A})$ & $42.85 \%$ \\
\hline D6 & 6 & 0 & 2 & 4 & $0(\mathrm{~A})$ & $33.33 \%$ \\
\hline $\mathrm{D} 7$ & 4 & 1 & 3 & 0 & $25 \%(\mathrm{~A})$ & $100 \%$ \\
\hline$\overline{\mathrm{D} 9}$ & 6 & 0 & 3 & 3 & $\begin{array}{c}0(\mathrm{~A}) \\
16.66 \%(\mathrm{I})\end{array}$ & $50 \%$ \\
\hline D10 & 5 & 0 & 4 & 1 & $0(\mathrm{~A})$ & $80 \%$ \\
\hline D11 & 3 & 0 & 1 & 2 & $0(\mathrm{~A})$ & $33.33 \%$ \\
\hline D12 & 5 & 1 & 4 & 0 & $\begin{array}{l}20 \%(\mathrm{~A}) \\
20 \%(\mathrm{I})\end{array}$ & $100 \%$ \\
\hline $\begin{array}{l}\mathrm{D} 13 \\
\end{array}$ & 5 & 1 & 3 & 1 & $20 \%(\mathrm{~A})$ & $80 \%$ \\
\hline$\overline{\mathrm{D} 14}$ & 5 & 0 & 3 & 2 & $0(\mathrm{~A})$ & $60 \%$ \\
\hline D15 & 6 & 1 & 4 & 1 & $\begin{array}{c}16.66 \%(\mathrm{~A}) \\
16.66 \%(\mathrm{I})\end{array}$ & $83.33 \%$ \\
\hline D17 & 3 & & & & $0(\mathrm{I})$ & \\
\hline D18 & 3 & $\overline{1}$ & 1 & 1 & $\begin{array}{c}33.33 \%(\mathrm{~A}) \\
33.33 \%(\mathrm{I})\end{array}$ & $66.66 \%$ \\
\hline D19 & 7 & 4 & 1 & 2 & $\begin{array}{c}57.14 \%(\mathrm{~A}) \\
42.8 \%(\mathrm{I})\end{array}$ & $71.42 \%$ \\
\hline D20 & 4 & 1 & 0 & 3 & $25 \%(\mathrm{~A})$ & $25 \%$ \\
\hline D21 & 5 & 0 & 2 & 3 & $\begin{array}{c}0(\mathrm{~A}) \\
20 \%(\mathrm{I})\end{array}$ & $40 \%$ \\
\hline$\overline{\mathrm{D} 22}$ & 4 & & & & $25 \%(\mathrm{I})$ & \\
\hline D23 & 6 & & & & $16.66 \%(\mathrm{I})$ & \\
\hline D24 & 4 & & & & $25 \%(\mathrm{I})$ & \\
\hline D25 & 4 & & & & $25 \%(\mathrm{I})$ & \\
\hline $\mathrm{D} 26$ & 3 & & & & $0 \%(\mathrm{I})$ & \\
\hline D27 & 3 & & & & $66.66 \%(\mathrm{I})$ & \\
\hline D28 & 26 & & & & $7.69 \%(\mathrm{I})$ & \\
\hline D29 & 5 & & & & $0(\mathrm{I})$ & \\
\hline D30 & 3 & & & & $0 \%(\mathrm{I})$ & \\
\hline D31 & 5 & & & & $20 \%(\mathrm{I})$ & \\
\hline D32 & 5 & & & & $40 \%(\mathrm{I})$ & \\
\hline Averages & & & & & $22.2 \%$ & $66.11 \%$ \\
\hline
\end{tabular}

Table 4: Summary of ASHG's HTML test results against the authors and INSPEC's results 


\begin{tabular}{|c|c|c|c|c|c|c|}
\hline \multirow{3}{*}{$\begin{array}{c}\text { Latex } \\
\text { Document }\end{array}$} & \multirow{3}{*}{$\begin{array}{c}\text { Number of } \\
\text { Subject Headings } \\
\text { generated by ASHG }\end{array}$} & \multirow{2}{*}{\multicolumn{3}{|c|}{ Author's Opinion }} & \multirow{3}{*}{$\begin{array}{l}\text { Accuracy } \\
\text { A: Author } \\
\text { I: INSPEC }\end{array}$} & \multirow{3}{*}{$\begin{array}{l}\text { OK/Good's } \\
\text { Accuracy }\end{array}$} \\
\hline & & & & & & \\
\hline & & Good & OK/Not Sure & Poor & & \\
\hline D1 & 5 & 2 & 3 & 0 & $\begin{array}{l}40 \%(\mathrm{~A}) \\
16.6 \%(\mathrm{I})\end{array}$ & $100 \%$ \\
\hline $\mathrm{D} 2$ & 5 & 4 & 0 & 1 & $66.66 \%(\mathrm{~A})$ & $66.66 \%$ \\
\hline D3 & 4 & 1 & 0 & 3 & $25 \%(\mathrm{~A})$ & $25 \%$ \\
\hline $\mathrm{D} 4$ & 10 from 11 & 4 & 4 & 2 & $40 \%(\mathrm{~A})$ & $80 \%$ \\
\hline D5 & 6 & 0 & 2 & 4 & $0(\mathrm{~A})$ & $33.33 \%$ \\
\hline D6 & 4 & 0 & 2 & 2 & $0(\mathrm{~A})$ & $50 \%$ \\
\hline D7 & 4 & 0 & 2 & 2 & $0(\mathrm{~A})$ & $50 \%$ \\
\hline D8 & 5 & 1 & 2 & 2 & $20 \%(\mathrm{~A})$ & $60 \%$ \\
\hline D9 & 4 from 6 & 0 & 2 & 2 & $\begin{array}{c}0(\mathrm{~A}) \\
25 \%(\mathrm{I})\end{array}$ & $50 \%$ \\
\hline $\mathrm{D} 10$ & 4 & 0 & 4 & 0 & $0(\mathrm{~A})$ & $100 \%$ \\
\hline D11 & 5 & 1 & 1 & 3 & $20 \%(\mathrm{~A})$ & $40 \%$ \\
\hline D12 & 5 & 0 & 5 & 0 & $\begin{array}{c}0(\mathrm{~A}) \\
20 \%(\mathrm{I}) \\
\end{array}$ & $100 \%$ \\
\hline D13 & 6 & 2 & 3 & 1 & $33.33 \%(\mathrm{~A})$ & $83.33 \%$ \\
\hline D14 & 4 & 0 & 4 & 0 & $0(\mathrm{~A})$ & $100 \%$ \\
\hline $\mathrm{D} 15$ & 5 & 1 & 4 & 0 & $\begin{array}{c}20 \%(\mathrm{~A}) \\
20 \%(\mathrm{I})\end{array}$ & $100 \%$ \\
\hline $\mathrm{D} 16$ & 4 & 1 & 2 & 1 & $25 \%(\mathrm{~A})$ & $75 \%$ \\
\hline D17 & 3 & & & & $0(\mathrm{I})$ & \\
\hline $\begin{array}{l}\mathrm{D} 18 \\
\end{array}$ & 3 & 1 & 1 & 1 & $\begin{array}{c}33.33 \%(\mathrm{~A}) \\
66.66 \%(\mathrm{I})\end{array}$ & $66.66 \%$ \\
\hline D19 & 3 & 1 & 0 & 2 & $\begin{array}{c}50 \%(\mathrm{~A}) \\
50 \%(\mathrm{I})\end{array}$ & $50 \%$ \\
\hline D20 & 4 & 1 & 0 & 3 & $25 \%(\mathrm{~A})$ & $25 \%$ \\
\hline $\mathrm{D} 21$ & 3 & 0 & 0 & 3 & $\begin{array}{c}0(\mathrm{~A}) \\
33.33 \%(\mathrm{I})\end{array}$ & 0 \\
\hline $\mathrm{D} 22$ & 7 & & & & $28.57 \%$ (I) & \\
\hline $\mathrm{D} 23$ & 7 & & & & $14.28 \%(\mathrm{I})$ & \\
\hline $\mathrm{D} 24$ & 4 & & & & $0(\mathrm{I})$ & \\
\hline $\mathrm{D} 25$ & 24 & & & & $25 \%(\mathrm{I})$ & \\
\hline $\mathrm{D} 26$ & 4 & & & & $0 \%(\mathrm{I})$ & \\
\hline $\mathrm{D} 27$ & 3 & & & & $66.66 \%(\mathrm{I})$ & \\
\hline $\mathrm{D} 28$ & 26 & & & & $50 \%(\mathrm{I})$ & \\
\hline D29 & 5 & & & & $0(\mathrm{I})$ & \\
\hline D30 & 4 & & & & $50 \%(\mathrm{I})$ & \\
\hline D31 & 25 & & & & $4 \%(\mathrm{I})$ & \\
\hline D32 & 4 & & & & $25 \%(\mathrm{I})$ & \\
\hline Averages & & & & & $22.91 \%$ & $62.75 \%$ \\
\hline
\end{tabular}

Table 5: Summary of ASHG's Latex test results against the authors and INSPEC's results 


\begin{tabular}{|c|c|c|c|c|c|c|}
\hline \multirow{3}{*}{$\begin{array}{c}\text { Text } \\
\text { Document }\end{array}$} & \multirow{3}{*}{$\begin{array}{c}\text { Number of } \\
\text { Subject Headings } \\
\text { generated by ASHG }\end{array}$} & \multirow{2}{*}{\multicolumn{3}{|c|}{ Author's Opinion }} & \multirow{3}{*}{$\begin{array}{l}\text { Accuracy } \\
\text { A: Author } \\
\text { I: INSPEC }\end{array}$} & \multirow{3}{*}{$\begin{array}{c}\text { OK/Good's } \\
\text { Accuracy }\end{array}$} \\
\hline & & & & & & \\
\hline & & Good & OK/Not Sure & Poor & & \\
\hline $\mathrm{D} 1$ & 5 & 2 & 3 & 0 & $\begin{array}{l}40 \%(\mathrm{~A}) \\
20 \%(\mathrm{I})\end{array}$ & $100 \%$ \\
\hline $\mathrm{D} 2$ & 17 & 1 & 3 & 13 & $5.88 \%(\mathrm{~A})$ & $23.52 \%$ \\
\hline D3 & 8 & 6 & 0 & 2 & $75 \%(\mathrm{~A})$ & $75 \%$ \\
\hline D4 & 4 & 1 & 2 & 1 & $25 \%(\mathrm{~A})$ & $75 \%$ \\
\hline D5 & 7 & 0 & 3 & 4 & $0(\mathrm{~A})$ & $42.87 \%$ \\
\hline D6 & 6 & 0 & 2 & 4 & $0(\mathrm{~A})$ & $33.33 \%$ \\
\hline D7 & 5 & 1 & 3 & 1 & $20 \%(\mathrm{~A})$ & $80 \%$ \\
\hline D8 & 5 & 2 & 2 & 1 & $40 \%(\mathrm{~A})$ & $80 \%$ \\
\hline D9 & 4 & 0 & 2 & 2 & $\begin{array}{c}0(\mathrm{~A}) \\
0(\mathrm{I})\end{array}$ & $50 \%$ \\
\hline D10 & 7 & 0 & 2 & 5 & $0(\mathrm{~A})$ & $28.57 \%$ \\
\hline D11 & 9 & 4 & 3 & 2 & $44.44 \%(\mathrm{~A})$ & $77.77 \%$ \\
\hline$\overline{\mathrm{D} 12}$ & 5 & 1 & 4 & 0 & $\begin{array}{c}20 \%(\mathrm{~A}) \\
20 \%(\mathrm{I})\end{array}$ & $100 \%$ \\
\hline D13 & 5 & 2 & 2 & 1 & $40 \%(\mathrm{~A})$ & $80 \%$ \\
\hline D14 & 7 & 0 & 3 & 4 & $0(\mathrm{~A})$ & $42.85 \%$ \\
\hline D15 & 4 & 1 & 3 & $\overline{0}$ & $\begin{array}{l}25 \%(\mathrm{~A}) \\
20 \%(\mathrm{I})\end{array}$ & $100 \%$ \\
\hline$\overline{D 16}$ & 7 & 3 & 1 & 3 & $42.85 \%(\mathrm{~A})$ & $57.14 \%$ \\
\hline D17 & 3 & & & & $0(\mathrm{I})$ & \\
\hline D18 & 3 & 1 & 0 & 2 & $\begin{array}{c}33.33 \%(\mathrm{~A}) \\
33.33 \%(\mathrm{I})\end{array}$ & $33.33 \%$ \\
\hline $\bar{D} 19$ & 5 & 1 & 1 & 3 & $\begin{array}{c}20 \%(\mathrm{~A}) \\
20 \%(\mathrm{I})\end{array}$ & $40 \%$ \\
\hline D20 & 5 & 1 & 0 & 4 & $20 \%(\mathrm{~A})$ & $20 \%$ \\
\hline $\bar{D} 21$ & 3 & $\overline{0}$ & $\overline{0}$ & 3 & $\begin{array}{c}0(\mathrm{~A}) \\
0 \%(\mathrm{I})\end{array}$ & 0 \\
\hline D22 & 4 & & & & $50 \%(\mathrm{I})$ & \\
\hline D23 & 8 & & & & $12.5 \%(\mathrm{I})$ & \\
\hline D24 & 4 & & & & $25 \%(\mathrm{I})$ & \\
\hline D25 & 28 & & & & $7.14 \%$ (I) & \\
\hline D26 & 4 & & & & $25 \%(\mathrm{I})$ & \\
\hline $\mathrm{D} 27$ & 44 & & & & $25 \%(\mathrm{I})$ & \\
\hline D28 & 4 & & & & $0(\mathrm{I})$ & \\
\hline D29 & 18 & & & & $27.77 \%(\mathrm{I})$ & \\
\hline D30 & 14 & & & & $14.28 \%(\mathrm{I})$ & \\
\hline D31 & 28 & & & & $14.28 \%(\mathrm{I})$ & \\
\hline D32 & 5 & & & & $40 \%(\mathrm{I})$ & \\
\hline Averages & & & & & $20.66 \%$ & $56.97 \%$ \\
\hline
\end{tabular}

Table 6: Summary of ASHG's Text test results against the authors and INSPEC's results 
After conducting the tests over the three document types, ASHG's average percentage accuracy was $21.92 \%$. Since our system was only based on the frequency and location of words in a document to determine the document's keywords and subject classification, it has missed the importance of the word senses and the relationship between words in a sentence. Our simplistic system did not capture the concepts behind the documents, or the ideas that the author is trying to convey. Our results support the idea that word frequency and location are not enough in information retrieval. However, since the ASHG's result will be used as a starting point by the author, he/she has the opportunity to correct the errors and include fields of the Semantic Header not given before registering it. Further work is required in refining the subject classification.

\subsection{Sample Result}

In this section, we will show an indexe that is generated by ASHG.

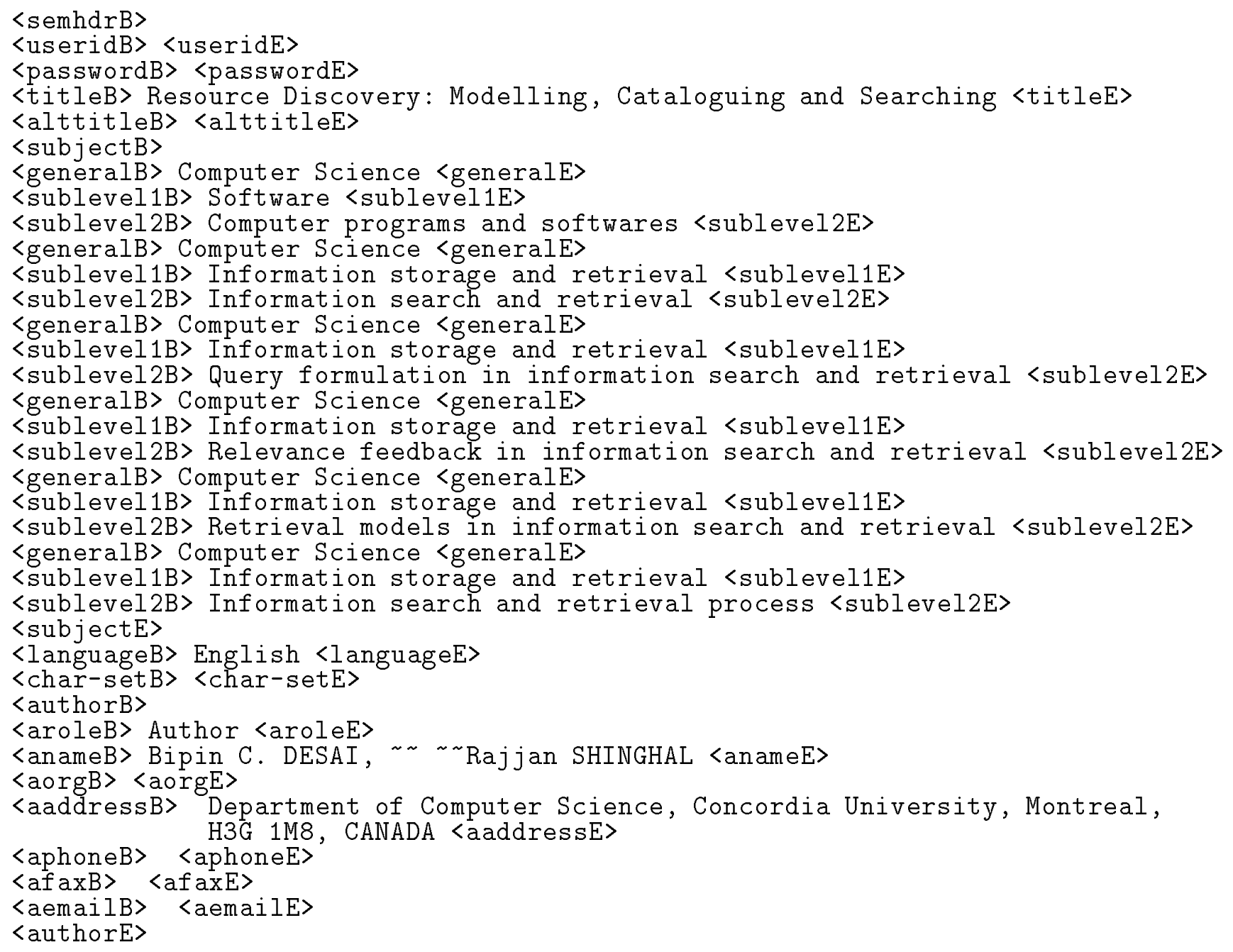




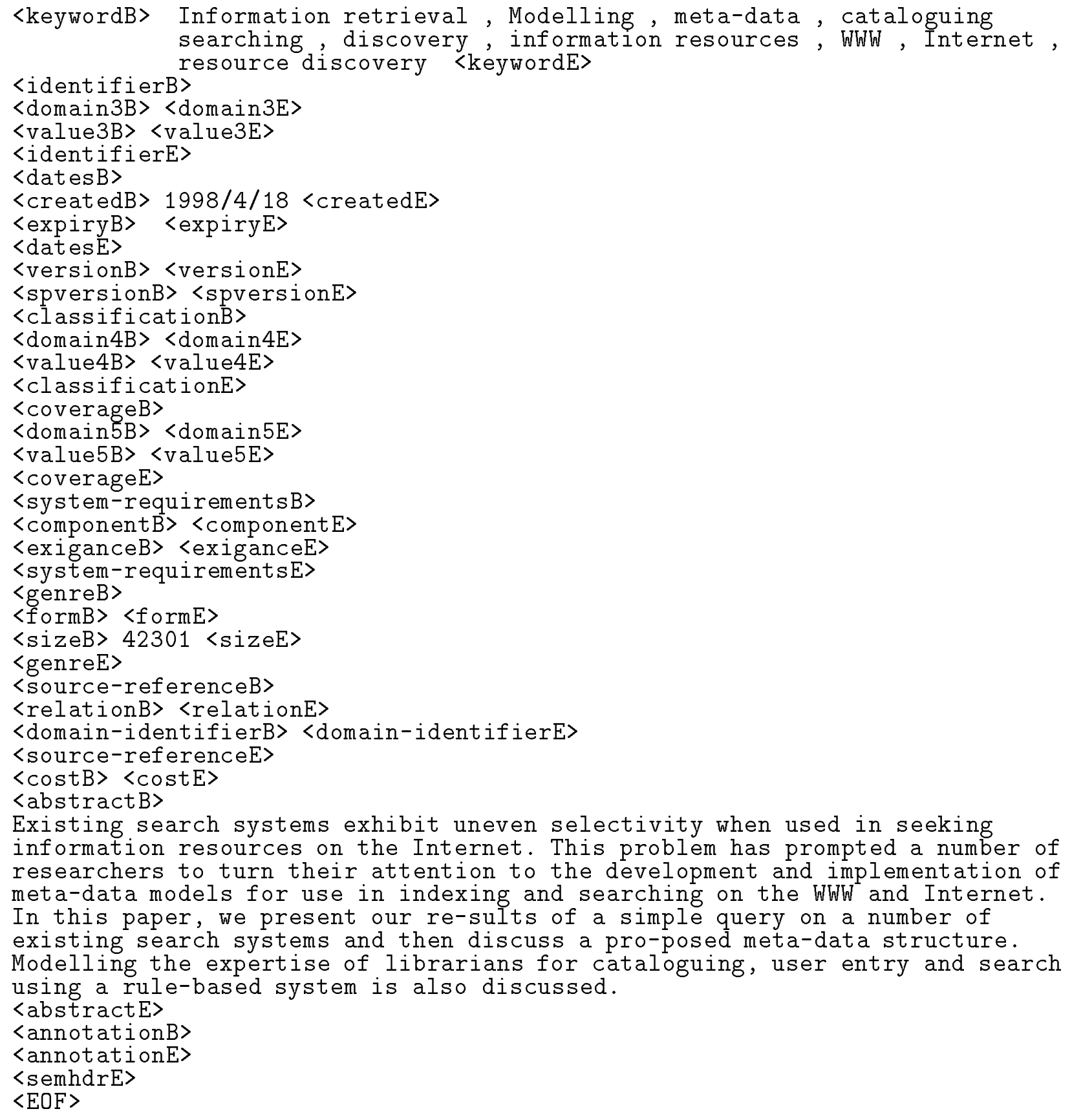

\section{Conclusion}

In this paper, we constructed CINDI's three level subject hierarchy for Computer Science and Electrical Engineering. CINDI's computer science subject hierarchy was based on ACM 
and CINDI's electrical engineering subject hierarchy was based on INSPEC. LCSH was used to augment both subject hierarchies. We also derived control terms from CINDI's subject headings. These control terms were associated with their subjects in CINDI's thesaurus. In addition, we presented a method of generating a Semantic Header, called ASHG. This scheme automatically extracts and generates an index or meta-information.

ASHG exploits the file naming conventions and the data within a document to determine the document's file type. ASHG exploits the semantics of the document's types in extracting the meta-information. It also applies automatic abstracting proposed by Luhn in generating document's abstract. It also assigns weights for terms depending on their location in the document. Both term weight and occurrence frequency were used in assigning terms for a document. These extracted terms were used to classify a document using the association between CINDI's controlled term and their subject headings in the thesaurus.

Finally, we applied ASHG to a collection of test documents and compared the results to the actual assignments made by INSPEC. We also consulted the papers'authors on ASHG's subject classification results. The results showed hundred percent accuracy in extracting the explicitly stated fields such as the title, abstract, author and keywords. They also showed some level of accuracy in generating the abstract.

Since our controlled terms were composed of terms found in CINDI's subject headings, ASHG's results showed a low degree of accuracy in classifying a document. The main reason was that some of the extracted terms were misleading. For example, the term wire should not be extracted unless it is followed by another term such as wire grid. The classification scheme used by ASHG showed some ineffectiveness, because it was based on term frequency and location information. For example, term-based retrieval cannot handle the following properties:

1. Different words may be used to convey the same meaning.

2. The same words may be used but they can have different meanings.

3. Different people may have different perspectives on the same single concept.

4. The same words may have different meanings in different domains.

Another weakness with ASHG is that it has not considered the issue of synonymity between words or between the subject headings.

In conclusion, we believe that resolving word senses and determining the relationships that those words have to one another will have the greatest impact on refining the ASHG's subject classification scheme. Therefore, the semantic level language processing should be handled by ASHG in the future. 


\section{Contribution of this Paper}

The contributions made by this paper to the CINDI project are listed below:

- An automatic subject hierarchy database builder was designed and built. The input to this builder is a subject headings of multiple levels. It produces a hierarchy of three levels. In addition, the subject hierarchy for both Computer science and Electrical engineering were constructed and derived from previously existing hierarchies such as ACM and INSPEC.

- A controlled term subject heading association was engineered. The paper used an existing spell program and built on it a stemming process that was used in relating the subject headings with their corresponding control terms.

- An automatic semantic header generator was designed and implemented. It extracted both implicit and explicit meta-information from the primary resource and it classifies it under a subject hierarchy. It handled HTML, Latex and Text documents.

\section{Future Work}

Some of the system's refinements should include:

- Terms, which are not significant alone, but are significant if they appear adjacent to another term should be extracted as significant terms. ASHG's keyword extraction process should handle more than single controlled terms. Future work should explore the effect of extracting noun phrases and compound controlled terms.

- Word senses and determining the relationships that those words have to each other should be resolved. The semantic level language processing should be handled by ASHG.

- The controlled terms and their synonyms should belong to the same control term and they should be associated with the same subject headings.

- The domain of the stop-word list should be explored, and more significant terms should be associated with the subject headings.

- Build more subject hierarchies such as Civil Engineering, Mechanical Engineering... Extend the type of documents that ASHG can extract meta-information from, such as RTF, SGML...

\section{References}

[1] Alvarado S., et al, Argument comprehension and retrieval for editorial text, Knowledge Based Systems 3 (3), pp. 139-162, 1990. 
[2] Andrews K., The development of a fast conflation algorithm for English, Dissertation submitted for the Diploma in Computer Science, University of Cambridge, 1971.

[3] Automatic indexing and classification of the WAIS databases: http://www.ub2.lu.se/autoclass.html.

[4] Baxendale P. B., Man made Index for Technical Literature - An Experiment, IBM Journal of Research and Development, 2:4, pp. 354-361, 1958.

[5] Belkin N., Croft W. B., Retrieval techniques, Annual review of information science and technology (ARIST), 22, pp. 109-145, 1987.

[6] Blair D. C. , Language representation in Information Retrieval, Elsevier Science publishers, New York, 1990.

[7] Brandow R. , Mitze K., Rau L. F., Automatic condensation of electronic publications by sentence selection, Information Processing and management, Vol. 31, No. 5., pp. 675-685, 1995.

[8] Chiaramella Y. et al., IOTA: a full text information retrieval system, In proceedings of the ACM conference on research and Development in information retrieval, edited F. Rabitti, Pisa, pp. 207-213, 1987.

[9] De Bra, P., Houben, G-J., \& Kornatzky, Y., Search in the World-Wide Web, http://www.win.tue.nl/help/doc/demo.ps

[10] Desai, B. C., An Introduction to Database Systems, West, St. Paul, MN 1990.

[11] Desai B. C., Cover page aka Semantic Header, http://www.cs.concordia.ca/faculty/bcdesai/semantic-header.html, July 1994, revised version, August 1994.

[12] Desai B. C., The Semantic Header Indexing and Searching on the internet, Department of Computer Science, Concordia University. Montreal, Canada, February 1995. http://www.cs.concordia.ca/ faculty/bcdesai/cindi-system-1.1.html

[13] DuRoss Liddy E., Anaphora in natural language processing and information retrieval, Information Processing and Management, Vol. 26, No. 1, pp. 39-52, 1990.

[14] Earl L. L., Experiments in Automatic Extracting and Indexing, Information Storage and Retrieval, 6:4, pp. 313-334, October 1970.

[15] Edmundson H. P., Problems in Automatic Abstracting, Communications of the ACM, 7:4, pp. 259-263, April 1964.

[16] Edmundson H. P. and Wyllys R. E., Automatic Abstracting and Indexing Survey and Recommendations, Communications of ACM, 4:5, pp. 226-234, May 1961.

[17] Edmundson H. P., New methods in Automatic Extracting, University of Maryland, college park, Maryland, Journal of the Association for computing machinery, Vol. 16, No. 2, pp. 264-285, April 1969. 
[18] Evans D. A. , Concept management in text via natural language processing: the CLARIT approach, In working notes for the AAAI spring symposium on Text-based intelligent systems. Stanford 1990.

[19] Fung R. and Del Favero B. , Applying Bayesian Networks to Information Retrieval, Communications of the ACM, Vol 38, No. 3, pp. 42-57, March 1995.

[20] Fletcher, J. 1993., Jumpstation, http://www.stir.ac.uk/jsbin/js

[21] Graham I., Introduction To HTML and URLs: http://www.utoronto.ca/webdocs/HTMLdocs/NewHTML/intro.html, Last Update: 24 January 1997.

[22] Hardy D. R., Shwartz M. F., Customized Information Extraction as a Basis for Resource Discovery, Department of Computer Science, University of Colorado. March 1994; Revised February 1995.

[23] Jacobs, P.S. and Rau, L.F., SCISOR: extracting information from online news, Communications of the ACM, Vol. 33, No. 11, 1990.

[24] Jacqueline W. T. Wong, W. K. Kan, Gilbert Young, ACTION: Automatic Classification for full-text documents, ACM Transactions on information systems, pp. 26-41, 1997.

[25] Jing Y. and Croft B. W., An Association Thesaurus for Information Retrieval, Department of Computer Science, University of Massachusetts at Amherst, Amherst, MA 01003.

[26] Johnson F. C., Paice C. D., Black W.J, Neal A. P., The application of linguistic processing to automatic abstract generation, Journal of Documentation and Text management, 1993.

[27] Katz, W. A., Introduction to Reference Work, Vol. 1-2 McGraw-Hill, New York, NY.

[28] Koster, M., ALIWEB(Archie Like Indexing the WEB), http://web.nexor.co.uk/aliweb/doc/aliweb.html

[29] Krovetz R., Croft W. B.. Lexical ambiguity and information retrieval, ACM Transactions on information systems, Vol. 10, No. 2, pp. 115-141, April 1992.

[30] Kupiec, J. , Pederson, J., and Chen F., A trainable document summarizer, In preceedings of the 18th ACMSGIR Conference, 1995.

[31] Lamport L., LATEX: A Document Preparation System, Addison_Wesley, Reading, Massachusetts, second edition, 1994, ISBN 0-201-52983-1.

[32] Lebowitz M., The use of memory in text processing, Communications of the ACM, Vol. 33, No. 8, pp. 30-49, 1990.

[33] Lehnert, W. G. and Sundheim, B. 1991. A Performance Evaluation of Text Analysis Technologies, AI Magazine 12(3):81-94. 
[34] Lewis D. D. , Jones K. , Natural Language processing for information Retrieval, Communications of the ACM, Vol 39, pp. 92-101, January 1996.

[35] Lovins J. B., Development of a Stemming Algorithm, Mechanical Translation and Computational Linguistics, Vol 11, January 1968.

[36] Luhn, H. P., The automatic creation of literature abstracts, IBM Journal of Research and Development, 2, pp. 159-165, 1958.

[37] Maron, M. E. and Kuhns, J. L., On relevance, probabilistic indexing and information retrieval, Journal of the ACM, 7, pp. 216-244, 1960.

[38] Mauldin M. 1991. Retrieval Performance in FERRET: A conceptual Information Retrieval System, In Proceedings, SGIR 1991. pp. 347-355.

[39] McBryan, Oliver A., World Wide Web Worm, http://www.cs.colorado.edu/home/mcbryan/WWWW.html

[40] O'Brien T., Oracle ConText, Text looms as the next frontier in Information Management, prepared by Oracle Corporation, April 1996.

[41] Oracle Corporation, ConText: Introduction to Oracle ConText, Oracle Corporation, Sept. 1993.

[42] Paice C. D., Automatic Generation of Literature Abstracts - An Approach Based on the identification of self indicating phrases, in information retrieval research, R.N. Oddy, S.E. Robertson, C.J. van Rijsbergen and P.W. Williams, editors, Butterworths, London, pp. 172-191, 1981.

[43] Paice C. D., Constructing Literature Abstracts by Computer: Techniques and Prospects, Information Processing and Management, 26:1, pp. 171-186, 1990.

[44] Porter, M. F., An Algorithm For Suffix Stripping, Program 14 (3), pp. 130-137, July 1980.

[45] Rau, L. F., Jacobs, P. S. and Zernik, U., Information extraction and text summarization using lingustic knowledge acquisition, Information processing and management, Vol. 25, No. 4, pp. 419-428, 1989.

[46] Rijsbergen C. J. van, Information Retrieval, second edition, Butterworths, pp. 17-22, 1979.

[47] Riloff E. and Hollar L., Text Database and Information Retrieval, ACM computing surveys, Vol. 28, No. 1, pp. 133-135, March 1996.

[48] Riloff E. and Lehnert W., Information Extraction as a basis for High-Precision Text Classification, ACM Transactions on Information Systems, Vol. 12, No. 3, pp. 296-333, July 1994.

[49] Rush J. E., Salvador R., and Zamora A., Automatic Abstracting and IndexingProduction of Indicative Abstracts By Application of Contextual Inference and Syntactic Coherence Criteria, Journal of the ASIS, 22:4,pp. 260-274, July-August 1964. 
[50] Salton G. and Lesk M. E., Computer Evaluation of Indexing and text processing, Journal of ACM, Vol 25, No. 1, pp. 8-36, 1968.

[51] Salton G., The SMART Retrieval System, Prentice-Hall Inc.,4-6, 1971.

[52] Salton G., McGILL M. J., Introduction to Modern Information Retrieval, McGraw-Hill Book Company, pp. 87-89, 1983.

[53] Salton G., Automatic Text Processing: The Transformation, Analysis, and Retrieval of information by Computer, Addison-Welsey, Reading, MA., 1989.

[54] Salton G., Allen J. , Buckley O. , Automatic Structuring and Retrieval of Large Text Files, Department of Computer Science, Cornell University. 1992.

[55] Salton G., Allan J., Buckley C., and Singhal A. , Automatic Analysis, Theme Generation, and Summarization of Machine-Readable Texts, Science, Vol264, pp. 1421-1426, June 1994.

[56] Shayan N., CINDI: Concordia INdexing and DIscovery system, Department of Computer Science, Concordia University, Montreal, Canada, 1997.

[57] Smeaton A. F., Progress in the Application of Natural Language Processing to Information Retrieval tasks, The Computer Journal, Vol. 35, No. 3, pp. 268-271, 1992.

[58] Stiles, H. F., The association factor in information retrieval, Journal of the ACM, 8, pp. 271-279, 1961.

[59] Teufel S. and Moens M., Sentence extraction as a classification task, ACL/EACL'97, Intelligent Scalable Text Summarization, Workshop Program, JULY 11, 1997.

[60] Thau, R., SiteIndex Transducer, http://www.ai.mit.edu/tools/site-index.html

[61] Turtle H. R. and Croft, W. B., Efficient Probabilistic Inference for Text Retrieval, In Proceedings of RIAO 91. pp. 644-661, 1991.

[62] Computer and Control Abstracts, Produced by INSPEC, No. 10, October 1997.

[63] http://www.oracle.com.sg/products/oracle7/oracle7.3/html/conTxtDS.html.

[64] http://www.oracle.com.sg/products/oracle7/oracle7.3/html/context_seybold.html.

[65] Experimental Search Engine Meta-Index, http://www.ncsa.uiuc.edu/SDG/Software/Mosaic/Demo/metaindex.html

[66] Library of Congress Subject Headings, September 1996.

[67] http://www.acm.org/class/1998/ccs98.txt.

[68] Search WWW document full text, http://rbse.jsc.nasa.gov/eichmann/urlsearch.html 
[69] WebCrawler, http://www.biotech.washington.edu/WebCrawler/WebQuery.html

[70] World Wide Web Catalog,

http://cuiwww.unige.ch/cgi-bin/w3catalog

[71] http://web.soi.city.ac.uk/research/cisr/okapi/stem.html

[72] http://www.qpat.com/info/help/stemhelp.html 